NBER WORKING PAPER SERIES

\title{
HOW WELL DO AUTOMATED LINKING METHODS PERFORM? LESSONS FROM U.S. HISTORICAL DATA
}

\author{
Martha Bailey \\ Connor Cole \\ Morgan Henderson \\ Catherine Massey \\ Working Paper 24019 \\ http://www.nber.org/papers/w24019 \\ NATIONAL BUREAU OF ECONOMIC RESEARCH \\ 1050 Massachusetts Avenue \\ Cambridge, MA 02138
}

November 2017, Revised May 2019

\begin{abstract}
Previously circulated as "How Well Do Automated Methods Perform in Historical Samples? Evidence from New Ground Truth." This project was generously supported by the National Science Foundation (SMA 1539228), the National Institute on Aging (R21 AG05691201), the University of Michigan Population Studies Center Small Grants (R24 HD041028), the Michigan Center for the Demography of Aging (MiCDA, P30 AG012846-21), the University of Michigan Associate Professor Fund, and the Michigan Institute on Research and Teaching in Economics (MITRE). We gratefully acknowledge the use the Population Studies Center's services and facilities at the University of Michigan (R24 HD041028). During work on this project, Cole was supported by the NICHD (T32 HD0007339) as a UM Population Studies Center Trainee. We are grateful to Ran Abramitzky, Eytan Adar, George Alter, Jeremy Atack, Hoyt Bleakley, Leah Boustan, John Bound, Charlie Brown, Matias Cattaneo, William Collins, Dora Costa, Shari Eli, Katherine Erickson, James Feigenbaum, Joseph Ferrie, Katie Genadek, Tim Guinanne, Mary Hansen, Kris Inwood, Maggie Levenstein, Bhash Mazumder, Jorgen Modalsli, Adriana LlerasMuney, Jared Murray, Joseph Price, Paul Rhode, Evan Roberts, Steve Ruggles, and Mel Stephens for their many helpful suggestions. We thank Sarah Anderson, Garrett Anstreicher, Ali Doxey, Meizi Li, Shariq Mohammed, Paul Mohnen, Mike Ricks, and Hanna Zlotnick for their many contributions to the LIFE-M project.
\end{abstract}

NBER working papers are circulated for discussion and comment purposes. They have not been peer-reviewed or been subject to the review by the NBER Board of Directors that accompanies official NBER publications.

(C) 2017 by Martha Bailey, Connor Cole, Morgan Henderson, and Catherine Massey. All rights reserved. Short sections of text, not to exceed two paragraphs, may be quoted without explicit permission provided that full credit, including $\odot$ notice, is given to the source. 
How Well Do Automated Linking Methods Perform? Lessons from U.S. Historical Data Martha Bailey, Connor Cole, Morgan Henderson, and Catherine Massey

NBER Working Paper No. 24019

November 2017, Revised May 2019

JEL No. J62,N0

\begin{abstract}
$\underline{\text { ABSTRACT }}$
This paper reviews the literature in historical record linkage in the U.S. and examines the performance of widely-used automated record linking algorithms in two high-quality historical datasets and one synthetic ground truth. Focusing on algorithms in current practice, our findings highlight the important effects of linking methods on data quality. We find that (1) no method (including hand-linking) consistently produces representative samples; (2) 15 to 37 percent of links chosen by prominent machine linking algorithms are identified as false links by human reviewers; and (3) these false links are systematically related to baseline sample characteristics, suggesting that machine algorithms may introduce complicated forms of bias into analyses. We find that prominent linking algorithms attenuate estimates of the intergenerational income elasticity by up to 20 percent and common variations in algorithm choices result in greater attenuation. These results recommend that current practice could be improved by placing more emphasis on reducing false links and less emphasis on increasing match rates. We conclude with constructive suggestions for reducing linking errors and directions for future research.
\end{abstract}

Martha Bailey

University of Michigan

Department of Economics

611 Tappan Street

207 Lorch Hall

Ann Arbor, MI 48109-1220

and NBER

baileymj@umich.edu

Connor Cole

University of Michigan

Department of Economics

611 Tappan Street

Ann Arbor, MI 48109

colecp@umich.edu
Morgan Henderson

University of Michigan

Department of Economics

611 Tappan St

Ann Arbor, MI 48103

morghend@umich.edu

Catherine Massey

Institute for Social Research

University of Michigan

426 Thompson Street

Ann Arbor, MI 48104

cgmassey@umich.edu 
New large-scale linked data are revolutionizing empirical social science. Record linkage is increasingly popular as a tool to create or enhance data for observational studies, randomized control trials, and lab and field experiments. Examples abound across subfields in economics, including health economics and medicine, industrial organization, development economics, criminal justice, political economy, macroeconomics, and economic history. In addition, current U.S. data infrastructure projects are linking national surveys, administrative data, and research samples to recently digitized historical records, such as the full-count 1880 (Ruggles et al. 2015, Ruggles 2006) and 1940 U.S. Censuses (the first Census to ask about education and wage income). ${ }^{1}$ These newly available "big data" have the potential to break new ground on old questions and open entirely novel areas of inquiry.

Automated machine-linkage methods are critical to many of these projects, especially the projects linking U.S. Censuses. However, outside of protected administrative and proprietary data enclaves, little is known about how machine linkage influences data quality and inference. This gap in knowledge reflects the lack of "ground truth" data to study false matches (Type I errors) and missed matches (Type II errors). ${ }^{2}$ Although some diagnostic exercises are suggestive, they rely on selected and often non-U.S. samples (Goeken et al. 2017, Christen and Goiser 2007, Eriksson 2016) or rich administrative data unavailable to most researchers (Scheuren and Winkler 1993, Winkler 2006, Massey 2017, Abowd 2017). The resulting uncertainty about the quality of machine-linked data may limit their value to social science.

This paper reviews the literature in historical record-linkage for the U.S. We then use two different historical U.S. samples to provide a novel evaluation of the effects of different linking algorithms on data quality and inference. Unlike contemporary data, historical data are public and contain identifiable

\footnotetext{
${ }^{1}$ Many on-going initiatives link the 1940 U.S. Census to other datasets. The Census Bureau plans to link the 1940 Census to current administrative and Census data (Census Longitudinal Infrastructure Project, CLIP) and the Minnesota Population Center plans to link it to other historical censuses. The Panel Survey of Income Dynamics and the Health and Retirement Survey are linking their respondents to the 1940 Census. The Longitudinal, Intergenerational Family Electronic Micro-Database Project (LIFE-M) is linking vital records to the 1940 Census (Bailey 2018). Supplementing these public infrastructure projects, entrepreneurial researchers have also combined large datasets. See, for example, Abramitzky, Boustan, and Eriksson (2012, 2013, 2014), Boustan, Kahn, and Rhode (2012), Mill (2013), Mill and Stein (2016), Hornbeck and Naidu (2014), Aizer et al. (2016), Bleakley and Ferrie (2013, 2017, 2016), Nix and Qian (2015), Collins and Wanamaker (2014, 2015, 2016), and Eli, Salisbury, and Shertzer (2018) . This paper discusses many of the linking approaches used in these papers.

2 "Ground truth" is defined as data obtained by direct observation of the true link.
} 
information, allowing us to be fully transparent about our samples and assumptions in assessing algorithm performance. Our samples include the Longitudinal Intergenerational Family Electronic Micro-database’s (LIFE-M) sample of birth certificates linked to the 1940 Census (Bailey 2018) as well as a sample of Union Army veterans from the Early Indicators Project genealogically linked to the 1900 Census (Costa et al. 2017). These data were linked by hand using multiple levels of review by highly trained individuals and, because they were created without knowledge of the machine links, do not preference one automated method over another.

However, even well-trained individuals and genealogists make errors. To examine the role of human error for our conclusions, we build a synthetic ground truth, which deliberately alters the data to mimic errors in the recording, transcription and digitization process. Although this synthetic ground truth is an imperfect representation of the more complicated errors in original historical records, the dataset's construction means that there is complete certainty about incorrect and correct links. In all cases, our analyses with the synthetic data result in very similar findings to hand-linked records. Although humans make mistakes, but these errors are not driving the conclusions in this paper.

Focusing on prominent algorithms and assumptions in current practice, the results from hand-linked and synthetic data highlight the important effects of linking methods themselves on data quality. First, no linking method produces samples that are consistently representative of the linkable population, and the ways in which the data are not representative differ across methods and data sources. Second, automated linking algorithms result in high rates of links rejected by human review, ranging from 15 to 37 percent in prominent methods. We find similar patterns in our synthetic ground truth data, which suggests links rejected in human review are, indeed, Type I errors. Third, different algorithms induce different correlations between false links and baseline sample characteristics, reinforcing concerns that the linking process itself may induce unpredictable forms of bias.

We also vary algorithm assumptions to offer insight into their impact on the quality of linked samples. We find that common uses of spelling standardization in deterministic algorithms tend to increase both Type I errors, from 16 to 60 percent, and Type II errors. Linking more common names using the same 
algorithms dramatically increases the share of links rejected in human review, although the share of links missed falls. Lastly, including records with exact ties on name, age, and birth place (often used in conjunction with simple probability weights) further increases error rates by 55 to 79 percent.

A case study illustrates the potential consequences of linking algorithms on inference. After characterizing the theoretical implications of linking errors in a bivariate setting using a within-between decomposition framework, we link the same fathers and sons to the 1940 Census using different machine algorithms and examine how the algorithms impact estimates of intergenerational mobility. We find that prominent linking algorithms themselves attenuate intergenerational income elasticity estimates by up to 20 percent. Frequently used variations on these methods, such as including more common names and phonetic name cleaning, result in attenuation upwards of 50 percent. Although using different linking methods results in different samples, eliminating false matches renders intergenerational income elasticities from different algorithms statistically indistinguishable. In our case study, Type I errors appear to be more important than sample composition (due to Type II errors) - a finding that cautions against efforts to increase match rates that come at the expense of increases in linking error.

These findings suggest that machine-linking algorithms per se can dramatically affect researchers’ conclusions. Different linking algorithms and assumptions induce different kinds of non-representativeness and measurement error, which may have unknown consequences for inference that are difficult to remedy. We conclude with easy-to-implement and low cost recommendations for improving machine linking and inference with linked samples. In particular, we recommend reweighting to address sample nonrepresentativeness and using hand-trained data and more data features to break ties among records, identify error rates, and train machine algorithms.

\section{THE EVOLUTION OF U.S. HISTORICAL RECORD LINKAGE}

Before the explosion of recent interest, record linkage has been a mainstay of social science for over 80 years. The earliest methods used painstaking manual searchers to link hand-written manuscripts. Recent developments in digitized records, computational speed, and probabilistic linking techniques have 
expanded the possibilities for automated, or machine-based, record linkage. We briefly summarize this early literature, focusing on the components of this history that laid the groundwork for current automated linking with historical records. See Ruggles, Fitch, and Roberts (2018) for a more detailed history of the findings in this early literature.

One feature important to historical record linkage and modern automated matching is "blocking.” Blocking refers to the partition of a dataset into "blocks" (or clusters of records) using a record attribute (Michelson 2006). This technique limits the number of potential matches according to the blocking attribute, thereby improving computational efficiency and (ideally) maintaining accuracy. For instance, blocking on place of birth and sex means that a linking algorithm looking for Franklin Jones born in Kentucky would only search within the set of candidate matches of men born in that state.

Historical linkage has always used blocking techniques, primarily to increase the feasibility of manually linking samples across manuscripts. The earliest blocking methods involved identifying a group of individuals in a particular location (e.g., township, county, or state) in one census and manually searching for the same people within the same region (the block) in the subsequent census (Malin 1935, Curti 1959, Bogue 1963, Thernstrom 1964, Guest 1987). While making manual searching feasible, this blocking strategy missed those who relocated or changed names between census years, generating unrepresentative samples (Ruggles 2006). Accordingly, samples linked using these methods necessarily omitted the geographically mobile population.

The creation of digitized state population indexes facilitated refinements in blocking. ${ }^{3}$ In one such approach that improved on previous methods, Steckel (1988) drew a random sample of households with children at least 10 years old in a historical census. He then searched for the same household in the previous census using the birth state of the child to narrow the search. This technique was able to locate individuals who moved between the census years, but it restricted the sample of linked households to those with children surviving to age ten.

${ }^{3}$ A state “index" is a list of individuals living in a state at a point in time. 
Advances in computing allowed improvements in automated matching, effectively replacing the time-intensive human search with computer queries. Leveraging newly created national population indexes and Public Use Microdata Samples (PUMS), automated matching began incorporating more data elements in the linking process. An early example of this strategy was Atack, Bateman, and Gregson (1992)'s probabilistic matching software, called "PC Matchmaker.” PC Matchmaker transformed names using phonetic codes and allowed for user-specified blocking and weighting schemes. Atack (2004) used this software to create a linked sample between the agricultural and population censuses between 1850 and 1880. Ferrie (1996)'s approach, which we describe in more detail in the following sections, aimed to create large, representative linked U.S. Census samples and has since been embraced by the literature, forming the basis of prominent methods in use today.

Subsequent sections summarize more recent developments in this literature. Before turning to these details, we present a stylized historical linking problem. This example illustrates common challenges in historical linking which recent developments in the linking literature seek to addresses.

\section{CURRENT APPRoAches TO LiNKING HistoricAl DATA}

Linking records requires choosing linking variables, also called “features.” Modern administrative records typically have multiple high quality features (e.g., full legal name, Social Security Number, exact date of birth, address). Outside of restricted administrative enclaves, however, data may dictate the use of a more limited set of linking features which may contain errors. We study historical data which are typical of records often available to researchers and have the advantage of containing identifiable information, allowing transparent study of how limited data and data errors affect the quality of linked samples. Like many modern records, historical data have limited information on individuals and contain errors in the original and due to digitization.

As an example, consider the challenge of linking birth certificates to the 1940 U.S. Census. Researchers typically use "time-invariant" features to do linking in order to minimize concerns about selection bias and non-representativeness in linked samples (Ruggles 2006). In U.S. Census linking, these 
variables typically include first name, last name, age, birth state, race, and sex. ${ }^{4}$ These variables may not be time invariant in practice, however. Names may vary over time, either because Census enumerators misspelled names, the individual reported incorrectly, or the individual changed their name (perhaps using a middle name or nickname in place of the given name). For example, Goeken et al. (2017) document that in two enumerations of St. Louis in the 1880 Census, nearly 46 percent of first names are not exact matches, and the Early Indicators project notes that 11.5 percent of individuals in the Oldest Old sample have a shorter first name in pension records than in the original Civil War enlistment records (Costa et al. 2017).

Similar problems arise in reported age and birth state. The recording of age in the Census tends to reflect "age heaping," a common practice of rounding of ages to the nearest multiple of five (A'Hearn, Baten, and Crayen 2009, Hacker 2013). Thus, individuals observed on birth certificates may not report their expected age in 1940. In addition, birthplaces are often inaccurately recorded. For example, Goeken et al. (2017) show —in the aforementioned double enumeration of St. Louis - that 8 percent of reported birthplaces do not match. In addition, rates of disagreement for mothers' and fathers' birthplaces for the same individuals are higher at 19 and 18 percent, respectively.

Compounding these recording errors, the digitization of hand-written manuscripts introduces the possibility that—even if variables were accurately written in the original—-they may still appear with error in the electronic records used for linking. For instance, our comparison of two independently digitized versions of the 1940 Census by Ancestry.com and FamilySearch.org suggests that 25 percent of records have different transcriptions of last name due to digitization alone.

These data quality issues are well known, and linking algorithms account for them by allowing for differences in age and name spelling across records. To deal with differences in age, researchers typically search over a range of ages. To account for orthographic variations in names, researchers have used two

\footnotetext{
${ }^{4}$ Matching in historical settings in other countries often makes greater use of characteristics not available in U.S. data. Modalsli (2017) notes that in Norway before 1910 there is less first name variation and more flexible surname traditions than in the U.S. In addition, Norwegian censuses use 500 birthplaces (municipalities) for a population of under 2 million, whereas the U.S. Censuses identify birthplaces as 48 states and foreign countries for a much larger population ( 132 million residents in the 1940).
} 
main approaches. First, some use phonetic string cleaning algorithms to account for minor spelling differences, name Anglicization, and transcription errors. Soundex, for example, was developed in the early $20^{\text {th }}$ century to help create Census links, simplifying names into phonetic codes to facilitate record searches. For example, Soundex assigns the same code to similar sounding names like "Smith," "Smyth" and "Smythe” (in this example, S530). Another cleaning algorithm, NYSIIS, the New York State Identification and Intelligence System, was developed as an improvement to Soundex in 1970. NYSIIS uses different transformations, for example, changing names like "Wilhem” and "William” to "WALAN." While these cleaned strings allow researchers to identify many good candidate links, matching on them deterministically may treat distinct names as the same. For instance, implementations of NYSIIS may categorize John and James as perfect matches (Ruggles, Fitch, and Roberts 2018). A second strategy for dealing with orthographic differences is to use metrics such as Jaro-Winkler or Levenshtein to quantify the dissimilarity of two names.

Figure 1 illustrates how limited information and measurement error create challenges for matching records. The linking problem is depicted as two-dimensional scatter plots after limiting candidate matches to those with the same birth state and sex (i.e., blocking on birth state and sex, as is common in the literature). The x-axis captures the similarity between the name on record to be linked and the names of candidate links in the 1940 Census, a string similarity metric such as the Jaro-Winkler similarity score, which will equal 1 if the names are identical and less than 1 otherwise. ${ }^{5}$ The $y$-axis captures the difference in the age in 1940 implied by the birth certificate (which contains exact date of birth) and the reported age in the 1940 Census. A perfect match in ages occurs when the age difference is zero.

In this two-dimensional space in Figure 1, candidate links fall into one of four categories:

(M1) A perfect $(1,0)$, unique match in terms of name and age similarity (Figure 1A).

(M2) A single, similar match that is slightly different in terms of age, name, or both (Figure 1B).

\footnotetext{
${ }^{5}$ Jaro-Winkler similarity score adapts the Jaro (1989) string score, the minimum number of single-character transpositions required to change one string into another, to up-weight differences that occur at the beginning of the string. See Winkler (2006) for an overview.
} 
(M3) Many perfect $(1,0)$ matches, leading to problems with match disambiguation (Figure 1C).

(M4) Multiple similar matches that are slightly different in terms of age, name, or both (Figure 1D).

As we discuss, historical linking algorithms generally treat M1 cases as matches. However, methods differ in their treatment of candidates in the M2, M3, and M4 categories. To account for differences in age as in M2, researchers typically search within a band of \pm 3 or \pm 5 years. Prominent approaches to dealing with ties in categories M3 or M4 include random selection among equally likely (tied) candidates (Nix and Qian 2015), equal probability weighting of tied candidates (Bleakley and Ferrie 2016), or the use of a weighted combination of linking features to help disambiguate potential matches (Feigenbaum 2016, Abramitzky, Mill, and Pérez 2018). The next sections describe how commonly used linking methods function and how they address challenges posed by M2, M3, and M4 cases in historical data.

\section{A. Ferrie (1996)}

Ferrie’s (1996) path-breaking approach links men in the 1850 U.S. Census to men who were 10 years and older in the 1860 U.S. Census. Ferrie (1996) begins by selecting a sample of uncommon names from the 1850 Census. ${ }^{6}$ To correct for minor orthographic differences (category M2 above), Ferrie transforms last names using NYSIIS codes and also truncates the untransformed first name after the fourth letter. Ferrie then links his sample to the 1860 Census and eliminates candidate links that were not born in the same state and not living with the same family. Ferrie keeps all candidate links within a \pm 5 year difference in age (or "age band") and, if more than two links remained, Ferrie chooses the link with the smallest age difference. At the end of this process, Ferrie drops cases where two individuals from 1850 link to the same observation in $1860 .^{7}$ This process produces a linked sample of 4,938 men-9 percent of the male population in 1850, and 19 percent of the population of men with uncommon names. Ferrie's approach varies in his more recent work, and he has used smaller age ranges, different ways of parameterizing name

\footnotetext{
${ }^{6}$ Ferrie (1996) searched for 25,586 men in the 1860 Census whose surname and first name appeared ten or fewer times in 1850.

${ }^{7}$ Ferrie (1996) does not specify a process for multiple match disambiguation - in his linking from 1850-1860, there were no ties after minimizing the difference in age.
} 
dissimilarities like SPEDIS, or altered restrictions on common names. More than 20 years later, Ferrie's original approach has become the foundation for much of the historical linking literature.

Two features of this algorithm are especially worth noting. First, the decision to make links among observations with uncommon names reduces both the computational burden and the number of candidate matches of the variety in M3. Consequently, this method never attempts to link common names like "John Smith.” Second, the decision to use NYSIIS and truncate first name may reduce problems associated with minor orthographic differences, but it may also increase ties of the variety in M3 and, therefore, the number of records the algorithm will not link. The individual effects of both of these features of the algorithm are considered in the analysis below.

\section{B. Abramitzky, Boustan, and Eriksson (2012 and 2014)}

Abramitzky, Boustan, and Eriksson’s (2012, 2014) “Iterative Method” automates Ferrie (1996) to use the full-count census. This procedure relaxes Ferrie's (1996) uncommon name restriction to the extent that the combination of name and age provide distinctive information. Summarized in a detailed web appendix, Abramitzky, Boustan, and Eriksson (2012) select a sample of boys ages 3 to 15 with unique name-age combinations in the 1865 Norwegian Census, standardize first and last names using NYSIIS codes, and look for exact, unique matches in U.S. and Norwegian Censuses. For the observations in the 1865 Census without an exact, unique link (M1), the algorithm then searches for a name match within a \pm 1 age band and, if there is no match in this band, the algorithm searches within a \pm 2 age band. The algorithm does not link a record if more than one candidate match exists within an age band. Abramitzky, Boustan, and Eriksson (2012) ultimately link a sample of 2,613 migrants and 17,833 non-migrants from a primary sample of 71,644 individuals for a match rate of 29 percent. Abramitzky, Boustan, and Eriksson (2014) use the same procedure to link men ages 18 to 35 with unique age-name combinations from the 1900 U.S. Census to the 1910 and 1920 Censuses, producing a sample of 20,225 immigrant and 1,650 native-born men for a match rate of 12 percent and 16 percent, respectively.

The authors provide the most recent version of their code for our analysis, which has been used for 
record linkage in a number of high profile papers. ${ }^{8}$ A variation on this approach is also reported in Abramitzky, Boustan, and Eriksson (2014)’s appendix as a robustness check. Similar to Ferrie’s (1996) uncommon name restriction, this variation requires that names be unique within a five-year age band in both Censuses.

Two differences to Ferrie (1996) are worth noting. First, as previously observed, Abramitzky, Boustan and Erickson (2012, 2014) link more common names while Ferrie’s (1996) algorithm does not. Second, Abramitzky, Boustan and Erickson (2012, 2014) use a narrower age band than Ferrie (1996).

\section{Feigenbaum (2016) and IPUMS (2015)}

A common feature of Ferrie (1996) and Abramitzky, Boustan, and Eriksson (2014) is that they search along the line segment where the phonetically cleaned name is identical with the candidate link (Figure 1, name string similarity $=1$ ) for a unique match in NYSIIS-cleaned name with a minimum age difference. This restriction reduces the computational burden substantially, but comes at the cost of excluding very similar (though not exact) names with exact or very close age matches. New methods in probabilistic linking extend these methods to create machine learning models that weigh different kinds of disagreements in names and ages. ${ }^{9}$ The key insight of this approach is that the best link may not exactly match on name (or phonetically cleaned name) or age as in Figure 1B and Figure 1D but it may dominate when simultaneously considering both age and name differences. Thus, this approach allows algorithms to

\footnotetext{
8 Many other papers have used variations on Ferrie (1996) and Abramitzky et al. (2014). These variations are similar in that they require matches to match completely on a cleaned name variable. For example, Abramitzky et al. (2013) use the Abramitzky et al. (2014) algorithm to match men aged 3 to 15 in the 1865 Norwegian Census to the 1880 U.S. and Norwegian Censuses, and match 26 percent of records unique by name and birth year from the 1865 Census. Boustan et al. (2012) link the IPUMS sample of the 1920 U.S. Census to the 1930 U.S. Census using a link uniqueness age band that functions similar to a uncommon names restriction, and match 24 percent of men unique by name, age and birthplace in their 1920 U.S. sample. This same dataset was used in Hornbeck and Naidu (2014). Eli, Salisbury, and Shertzer (2018) match Civil War recruitment records from Kentucky to U.S. Censuses before and after the Civil War using a variation of Ferrie (1996) without an uncommon names restriction, and impose an additional restriction on Jaro-Winkler string dissimilarity after generating candidate matches with NYSIIS. They match 30 percent of selected records of recruits from the 1860 U.S. Census to the 1880 U.S. Census. Aizer et al. (2016) match state mother's pension records to other data sources, including the Social Security Death Master File, using a variation on Ferrie (1996) without an uncommon names restriction and Soundex, but allow some additional matches to differ in exact name but have low SPEDIS and Levenstein dissimilarity measures. Aizer et al. (2016) match 48 percent of their records to the Social Security Death Master File, but this high match rate reflects the fact that they can use exact date of birth to match their observations.

${ }^{9}$ See Mullainathan and Spiess (2017) for a useful primer on machine learning for economists.
} 
resolve some cases that deterministic algorithms like Ferrie (1996) and Abramitzky, Boustan and Erickson (2014) cannot.

One class of machine learning algorithms, known as "supervised learning," use training data to classify matches. Training data may be a subset of data coded by humans (sometimes genealogists and sometimes by others, e.g., “clerical review”) or by observation of true links (called ground truth). If the training data are ground truth and the model is well specified, the computer will learn how to classify links to approximate this truth. However, if the training data are of limited quality (e.g., humans make many erroneous decisions), the computer algorithm will replicate these incorrect decisions. Another problem that might occur for ground truth or human links is that, if the training data have little in common with the records to be linked, the supervised-learning algorithm will not learn as well, and its performance will be unpredictable. In short, the performance of supervised learning algorithms depends heavily on the quality of the training data as well as how similar the training data are to the data to be machine-linked.

The Minnesota Population Center (MPC) used a supervised learning method as part of its largescale linking of the Integrated Public Use Microdata Series Linked Representative Samples (IPUMS-LRS), a set of links between the 1850 to 1930 one-percent samples and the 1880 Census (Ruggles et al. 2015). Using clerically reviewed data, MPC trains a Support Vector Machine (SVM) using features of matches that, after specifying a few tuning parameter choices, classifies links as true or false (Goeken et al. 2011). Illustrating the conservative nature of their approach, they produce final match rates of 12 percent for native-born whites, 3 percent for foreign-born whites, and 6 percent for African Americans for the 18701880 links. ${ }^{10}$ Unfortunately, this model is proprietary and we cannot use it in our analysis.

In a similar spirit, Feigenbaum (2016) uses a different supervised-learning technique to link the 1915 Iowa state Census to the 1940 U.S. Census. After creating training data by hand, he estimates a probit model for whether or not a potential link is linked on a variety of record features, including name Jaro-

\footnotetext{
${ }^{10}$ Researchers have used the IPUMS-LRS for a variety of research questions, including the economic effects of racial fluidity (Saperstein and Gullickson 2013), long-term differences in black and white women's labor-force participation (Boustan and Collins 2014), and intergenerational co-residency (Ruggles 2011).
} 
Winkler similarity scores, differences in age, indicators for Soundex matches of first or last name, indicators for matches in letters or names, and indicators for matching truncated first or last names. He then tunes his model so that a link is only chosen if its estimated probability of being a match is sufficiently high and sufficiently greater than the second-best candidate’s match probability (if a second-best candidate exists). He chooses the parameters using their performance offs in the training data. Feigenbaum (2016) achieves a match rate of 57 percent. $^{11}$

\section{Abramitzky, Mill, and Pérez (2018)}

An alternative to supervised learning techniques is unsupervised learning, an approach which evaluates quality of different links without using training data. Instead, these algorithms depend on using observed patterns in features of the data to fit models that separate the data by quality of potential link. One advantage of the unsupervised learning approach is that it allows machine linking in contexts without training data. Similar to other deterministic methods like Ferrie (1996) and Abramitzky, Boustan, and Eriksson (2014), this method is replicable and consistent. In contrast, supervised learning algorithms depend on training data which may not be available. Moreover, if training data are error ridden or too different from the dataset to be linked, the lack of reliance on them is a feature. Not using human decisions, however, means that the algorithm's performance depends on difficult-to-validate modelling assumptions.

Abramitzky, Mill, and Pérez (2018) use unsupervised learning in the form of the expectationmaximization algorithm (Fellegi and Sunter 1969, Winkler 2006, Dempster, Laird, and Rubin 1977) to link Censuses in the U.S. and Norway. Building on Mill (2013), they fit a mixture model that allows for conditionally independent multinomial probabilities of specific age distances and discretized Jaro-Winkler

\footnotetext{
${ }^{11}$ We focus on Feigenbaum (2016) in this analysis because it was developed for U.S. data, and is transparent and easy to replicate. Many other researchers have also incorporated probabilistic and machine learning. Mill (2013) and Mill and Stein (2016) use an expectation maximization method. Similar to the IPUMS-LRS, Wisselgren et al. (2014) use a support vector machine and link the 1890 Swedish Census to the 1900 Swedish Census in a few select parishes for match rates ranging from 25 to 72 percent. Antonie et al. (2014) link across historical Canadian census data and achieve linkage rates from 17.5 percent (Quebec) to 25.5 percent (New Brunswick). Other work uses Ancestry.com's search algorithm to link records. Bailey et al. (2011) link records of lynching to the 1900 to 1930 U.S. Censuses using the Ancestry.com's algorithm, linking 45 percent of their lynching records. Collins and Wanamaker (2014) and (2015) search Ancestry using Soundex for names as well as age and place of birth for white and black men ages 0 to 40 resident in Southern states in the 1910 Census sample. They match 19 and 24 percent of men, respectively, to a unique person in 1930.
} 
scores for two distributions separately and fit this model with observed data using the expectationmaximization algorithm. Then, using their results, they calculate the estimated probability that a given potential match is a correct match conditional on the Jaro-Winkler score and age distance of the match. Like Feigenbaum (2016), they create a final set of matches by applying cut-offs to these estimated probabilities so that links are chosen that reach a sufficiently high estimated probability that is sufficiently greater than the second-best candidate match. However, unlike Feigenbaum (2016), these cutoffs are not guided by performance of the algorithm in a test or validation sample as the method is designed for cases where no training data exist.

The approach is not completely automated, because it requires the user to define other parameters, including discretization thresholds and probability cut-offs for classifying a link. ${ }^{12}$ With regards to the latter, using lower cut-offs will create more matches but potentially include more marginal matches less likely to be correct. Conversely, higher cut-offs will create fewer matches but create matches that have a higher estimated probability of being correct. To address this trade-off, Abramitzky, Mill, and Pérez (2018) use two cut-offs, a more conservative and less conservative choice. Using these two cut-offs for their algorithm, they achieve match rates of 5 percent and 15 percent in their Census data. Our analysis implements these cutoffs and considers the effects of alternate cut-offs in an appendix.

In summary, existing linking methods involve multiple differing assumptions that may affect match rates but have unknown effects on linking errors. Which set of assumptions should researchers use in different contexts? What are the implications of using variations on these algorithms? This paper seeks to answer these questions by presenting a systematic comparison of methods in different records and periods.

\section{DATA AND METRICS OF AUtOMATEd METHOd PERformance}

This paper evaluates the performance of four different linking methods for linking U.S. historical

\footnotetext{
12 These choices may be consequential. For example, setting a high Jaro-Winkler similarity threshold corresponding to $(0.92,1]$ assigns the same estimated match probability to a pair with first names, Katherine/Catherine, as to a pair with an exact match on first name, all else equal.
} 
data: Ferrie (1996); Abramitzky (Abramitzky, Boustan, and Eriksson 2014); regression-based, supervised learning (Feigenbaum 2016); and unsupervised machine learning (Abramitzky, Mill, and Pérez 2018). Detailed web appendices, published articles, and posted code make replicating these methods straightforward. Ferrie (1996) and Feigenbaum (2016) describe their methods step by step, which we implement exactly as described. ${ }^{13}$ We present Feigenbaum (2016) using both his regression coefficients for the Iowa Census-1940 training data (labeled "Iowa coef.”) as well as coefficients estimated using handlinked samples (called "Estimated coef."; see Appendix A for details and coefficient estimates). To implement Abramitzky, Boustan, and Eriksson (2014) and Abramitzky, Mill, and Pérez (2018), we use the code provided by the authors (see Appendix A) and report two cutoff implementations per the latter's recommendation, "less conservative” and "more conservative." We additionally present results for the Ferrie (1996) and Abramitzky, Boustan, and Eriksson (2014) methods using varying assumptions about phonetic name cleaning, more common names, and methods for breaking ties.

\section{A. Hand-linked and Synthetic Data}

We examine the performance of each algorithm in two high-quality, hand-linked historical samples: the LIFE-M sample of birth certificates linked to the 1940 Census (Bailey 2018) and a genealogically linked sample of Union Army veterans from the Early Indicators Project (Costa et al. 2017).

The LIFE-M sample is based on a random draw from birth certificates from Ohio and North Carolina. These birth certificates are then linked to siblings' birth certificates using parents' names. We exclude girls because they typically changed their name at marriage in this era, making them hard to find as adults in the Census (see Appendix B). The LIFE-M sample consists of 42,869 boys born from 1881 to 1940, 24,408 of whom were born in North Carolina and 18,461 born in Ohio.

The LIFE-M sample of boys is then linked to the 1940 full-count U.S. Census using a semi-

\footnotetext{
${ }^{13}$ Unlike Ferrie (1996), we do not limit links based on family continuity. In addition, we treat records with multiple matches after the last step as having no link, although Ferrie reports having none of these instances and, therefore, does not indicate how he would have dealt with them.
} 
automated process, making use of both computer programming and human input. Our linking variables include first, middle (when available), and last name, birth state, and age. We do not use race, because it is not available on all birth certificates (see section V.B for an analysis of linking implications).

After cleaning and standardizing the data, we use bi-gram matching on name and age similarity within birth state to generate a set of candidate links (Wasi 2014).${ }^{14}$ Each candidate match is reviewed by two "data trainers" who choose a correct link (or no link) from the set of candidates. If the two trainers agree, we treat their choice (link or no link) as the truth. In cases where the two trainers disagree, the records are re-reviewed by three new trainers to resolve these discrepancies (see section III.B.3). ${ }^{15}$

LIFE-M data trainers are taught to reject links if they are not completely certain the links are correct. Before they are allowed to train data independently, trainer decisions must reach a 0.95 correlation with a truth dataset—a learning process requiring working with a senior data trainer and multiple rounds of feedback across approximately 30 hours of work. After trainers achieve this 95-percent threshold, an automated system distributes training batches to trainers, guaranteeing that links are reviewed by two different trainers initially and that discrepancies are reviewed by three additional trainers. This automated system also distributes audit batches (that are indistinguishable from training work) at least once per week to provide weekly feedback to trainers and monitor accuracy. Trainers meet weekly to discuss their mistakes, difficult cases, and learn about historical-contextual factors affecting the quality of the data.

The Family History and Technology Lab at Brigham Young University (BYU) performed two independent quality checks of the LIFE-M links. First, BYU research assistants used genealogical methods and multiple data sources to hand link 543 of the 18,461 Ohio boys, 241 of which had been linked by LIFEM. The BYU team had no knowledge of LIFE-M’s links. Among links made by both LIFE-M and BYU, BYU agreed with LIFE-M matches 93.4 percent of the time (16/241 matches were discordant). Second,

\footnotetext{
${ }^{14}$ We generate a set of candidate links using “reclink.ado”, an algorithm that uses bi-gram comparisons of name strings. We also block on the first letter of last names to reduce computation time.

15 "Data trainers" participate in a rigorous orientation process where they receive detailed feedback on their accuracy relative to an answer key. They continue this process for 10 to 20 hours per week until their matches agree with the truth dataset 95 percent of the time. After completing this orientation, trainers become part of the larger team that conducts independent clerical review.
} 
BYU compared 1,043 LIFE-M links to those already on the FamilySearch.org “Tree.” (FamilySearch.org Tree links are created by genealogists and users of FamilySearch.org, which are also independent of the LIFE-M process.) For 1,043 birth certificates linked to the 1940 Census by LIFE-M and FamilySearch.org users, the LIFE-M links agreed with FamilySearch.org users 96.7 percent of the time. Taking genealogical linking as the gold standard implies that LIFE-M’s false link rate is between 3.3 and 6.6 percent. To account for potential errors in the LIFE-M data, we additionally require all links that differ from the LIFE-M sample to be re-reviewed using the "police line-up” process as described below.

Our second sample is the Oldest Old sample of Union Army veterans from the Early Indicators Project. Costa et al. (2017) created this sample of 2,076 individuals at least 95 years old linked to the 1900 complete-count U.S. Census using genealogical methods and a rich set of supplementary information. These veterans tended to report complete and accurate information to ensure they would receive their army pensions and benefits. Moreover, sources such as gravestone databases, obituaries, newspaper accounts, veterans associations and pension files allow multiple cross-validation exercises, ultimately resulting in a high match rate of 90 percent among men confirmed to live beyond the 1900 Census. The Early Indicators Project scores matches on a scale of 1 to 4 to indicate their confidence in a match. We use 1,887 matches coded as the highest quality (1 and 2) as the hand-linked sample. Importantly, we do not use all possible records for which matches were attempted.

Because these hand-linked data may contain errors, we validate our conclusions by building a third sample: a synthetic ground truth. This synthetic ground truth adds noise to true links to mimic errors in historical data while ensuring complete certainty about correct and incorrect links. That is, this synthetic dataset characterizes the performance of each matching algorithm relative to an objective truth, which shares important data features with the LIFE-M sample.

We construct the synthetic ground truth in two steps. First, we take all of our Ohio and North Carolina born boys' birth certificates, randomly drop 10 percent to reflect mortality and emigration and 
drop another 5 percent to reflect under-enumeration. ${ }^{16}$ Using the LIFE-M records as a basis allows us to retain sample name characteristics (e.g., ethnic origin and other conventions and name commonness). To account for orthographic differences in enumeration or transcription errors, we add noise to names and ages to reflect age heaping and transcription or digitization errors (Goeken et al. 2017, Hacker 2013, 2010). ${ }^{17}$ One limitation of this approach is that the true error structure in names and ages is unknown, so our decisions about how to simulate error may be simplistic and incomplete.

The resulting synthetic truth dataset is a noisy version of the truth for 85 percent of the Ohio and North Carolina boys. Then, we append to a random sample of boys from the 1940 Census who were born in Michigan, Indiana, Tennessee and South Carolina. Because these states neighbor Ohio and North Carolina, these individuals are incorrect links by construction. We chose these states because they retain regional naming conventions and have similar demographic and economic characteristics and, consequently, provide good candidate links. We choose the size of our random sample of boys from neighboring states so that our total set of candidate matches for each state has the same number of observations as in the LIFE-M linking exercise: 3,133,982 boys from the relevant age ranges born in Michigan and Indiana for Ohio and 1,904,592 boys born in Tennessee or South Carolina for North Carolina. We then use the algorithms to link between the unaltered birth certificates and this set of simulated candidate matches. When linking to this dataset, we emulate the common process of blocking on birthplace and consider only the synthetic Ohio data as candidate matches for the Ohio boys and only the synthetic

\footnotetext{
${ }^{16}$ Based on life tables from 1939 to 1941 , we calculate that 8 percent of our sample should be un-linkable due to death prior to 1940 (National Office of Vital Statistics 1948). Moreover, Census analyses estimate that around 5.4 percent of individuals were missed in 1940 (West and Robinson 1999). This calculation leaves some scope (about 1.5 percentage points) for emigration, which reflects the fact that we think emigration for native-born boys would have been much lower than for those born abroad. To the extent that our approximation of emigration is too low, the actual Type II errors should be adjusted accordingly.

${ }^{17}$ To mimic age-heaping, 25 percent of ages are rounded to the closest multiple of 5 . We introduce orthographic and transcription errors as follows. In 10 percent of cases, the first and middle names are transposed (if a middle name exists) and, in 5 percent of cases, the first and last names are transposed. In 5 percent of cases each, the first character of the first name or last name is randomly changed. In 5 percent of cases, each second character of the first name or last name is randomly changed. In 5 percent of cases, each third character of the first or last name is randomly changed. In 5 percent of cases each, we add a repeated letter to first names (e.g., "James" $\rightarrow$ "Jamees") or last names. In 5 percent of cases each, a random letter is dropped or two letters are transposed in the first or last name (e.g., "Matthew" $\rightarrow$ "Mathew" or "William” $\rightarrow$ "Willaim”). In 5 percent of cases, we replace the first name with an initial. In 50 percent of cases, we drop middle names (resulting in the same share of observations having middle names as is observed in the 1940 Census).
} 
North Carolina data as candidate matches for the North Carolina boys.

\section{B. Performance Criteria}

We use four main criteria to measure performance. The first two are almost universally reported in papers using linked samples.

(1) Match rate: We calculate the match rate as the share of records that were successfully matched from the sample that we attempted to link. Even for perfect matching, this rate is expected to be less than 100 percent due to emigration and mortality. Notwithstanding, comparisons across methods are still valid, as emigration and mortality affect each sample and, therefore, all of our methods equally.

(2) Representativeness: We compare a set of characteristics of the linked samples to the same characteristics of the unlinked sample using a heteroskedasticity-robust Wald test of the overall joint significance of the covariates in a multivariate, linear probability model with Huber-White standard errors (Huber 1967, White 1980). The exact variables in the regression vary across datasets. Under the null hypothesis that the covariates are jointly unrelated to successful linkage, the Wald-statistic should be statistically insignificant. ${ }^{18}$ This approach provides a straightforward, single summary measure, and its regression coefficients describe the extent the extent of non-representativeness and subgroups that are under-represented.

These measures are inadequate to assess link quality. This fact is easily illustrated in an example. Consider a matching algorithm that randomly links individuals between two datasets. This algorithm would perform very well in terms of the first two criteria, because the entire sample would be matched and identical to the baseline sample in observed characteristics (and, therefore, representative). Few researchers, however, would want to work with these data, because — with large enough datasets—-the incidence of false links would approach 100 percent, rendering the data useless for making population inferences.

\footnotetext{
${ }^{18}$ We implement this in Stata by multiplying the F-statistic reported in Stata following a regression with robust standard errors by the relevant degrees of freedom parameter. Note that this test could be very conservative in the sense that it would reject the null hypothesis due to one variable's significance in the regression and does not weight for the 'importance' of different covariates.
} 
We, therefore, use two more criteria to assess link performance (Abowd and Vilhuber 2005, Kim and Chambers 2012).

(3) False link rate (Type I error rate ${ }^{19}$ : We compare links for each automated method to a measure of the truth. We treat the high-quality, hand-linked Early Indicators dataset as the "truth," given that genealogists have used multiple data sources to confirm each link. In the synthetic data, we know the true link, so we code differences in links between an algorithm and the synthetic data as Type I errors.

For the LIFE-M data, however, we subject discrepancies between the hand-links and the automated methods to an additional blind review. Similar to a "police line-up,” two reviewers independently review the LIFE-M link (made by hand), the link made by the automated method, and close candidate links. Reviewers may choose to code any of these links as correct or incorrect. This process gives the links from the hand-match and the automated method an equal shot at being chosen to avoid preferential treatment. For the LIFE-M data, only links that are rejected in clerical review as part of the police line-up are treated as Type I errors. This analysis may understate the true Type I error rate if the hand-links are incorrect and agree with the automated method.

(4) False negative rate (Type II error rate) $)^{20}$ : This metric captures the fraction of true links that are not found, or 1 - Match Rate*(1 - Type I Error Rate). With this definition, the false negative rate can never be zero, because mortality and emigration mean that many individuals cannot be linked even with perfect data. $^{21}$

\section{The Performance of Prominent Automated Matching Methods}

Because a central focus of a growing literature is linking to the newly available 1940 Census, we begin our analysis with the LIFE-M sample to the 1940 Census. We then corroborate our findings using

\footnotetext{
${ }^{19}$ Computer scientists focus on precision, or 1-T1 error rate presented here.

${ }^{20}$ Computer science focuses on a similar statistic, "recall." This measure is defined as the number of true links found by the algorithm divided by number of linkable observations, or those linked by the data trainers.

${ }^{21}$ Note also that, if the marginal link is more likely to be incorrect, an increase in the match rate within a specific algorithm has a weakly negative effect on Type II error rates and a weakly positive effect on Type I error rates. If the marginal link is wrong, then the Type II error rate would not change but the Type I error rate would increase. However, if the marginal link is correct, the Type II error rate would fall and the Type I error rate would decrease.
} 
our synthetic ground truth and the Oldest Old sample from the Early Indicators Project.

\section{A. Evaluating Algorithms Using the LIFE-M Data}

Figure 2 compares the performance of selected, prominent automated linking methods to the handlinked LIFE-M data, where each of these methods uses the same information-name, age, and birth stateto create links. The length of each bar represents the match rate, computed as the share of the baseline sample of 42,869 boys who were successfully matched to the 1940 complete count Census. LIFE-M handreview matched 45 percent of the baseline sample. Ferrie's (1996) method matched 28 percent of the baseline sample, and Abramitzky, Boustan, and Eriksson (2014) achieve a higher link rate of 42 percent. This result makes sense because a central difference between the two methods is that Abramitzky, Boustan, and Eriksson (2014) do not impose Ferrie’s (1996) uncommon name restriction. Feigenbaum’s (2016) regression-based machine learning method matches 52 percent of the baseline sample both when using Iowa coefficients and when we estimate the coefficients using a random sample of the LIFE-M links. Abramitzky, Mill, and Pérez (2018)’s expectation-maximization method links 46 percent of the sample when using less conservative cutoffs and 28 percent of the sample with more conservative cutoffs.

Across the board, these match rates are higher than the match rates in the original studies. For instance, the Ferrie (1996) method matches 28 percent of the LIFE-M data versus his published figure of 9 percent of all men between 1850 and 1860 Censuses. Similarly, Abramitzky, Boustan, and Eriksson (2014) link 40 percent of the LIFE-M sample, whereas the same method links only 29 percent in Abramitzky, Boustan, and Eriksson (2012) and 16 percent of native-born men in Abramitzky, Boustan, and Eriksson (2014). These higher match rates may reflect the quality of the birth certificate data compared to other sources. Birth certificates (1) contain a complete and correct full name, often including middle names omitted in the Census; (2) record the exact date of birth rather than age in years; ${ }^{22}$ and (3) capture the birth state by construction (it is issued by the birth state and so should not have reporting error like the Census).

\footnotetext{
${ }^{22}$ Massey (2017) shows that decreasing the noise in age results in higher match rates and lower Type I error rates.
} 
Finally, the LIFE-M boys are on average 24 years old in the 1940 Census, so mortality and outmigration are likely lower for them than in other studies.

Figure 2 also summarizes the share of links that human reviewers rejected in a blinded review using the "police line-up” method described in section II. These rejected links are presented in two ways. First, the share of the entire sample determined to be wrong for each method is displayed in red in Figure 2. For less than 2 percent of original sample, trainers reversed LIFE-M decisions upon re-review in favor of the link chosen by one of the automated methods. Consistent with genealogical validation, these reversals are rare. Second, the column on the far right in Figure 2 presents that share of links that were rejected by human reviewers (the estimated Type I error). We compute this share by dividing the share of the total sample that is incorrect by the match rate. Because the LIFE-M match rate is 45 percent, this implies a Type I error rate of 4 percent (approximately 0.017/0.445). As we illustrate in section VI, the implications of measurement error for inference may be, in general, linked to the share of incorrect links, so our discussion focuses on this second metric.

Relative to clerical review, the share of false links for automated methods is higher across the board. The lowest Type I error rate occurs in the more conservative version of Abramitzky, Mill, and Pérez (2018) at 15 percent. Ferrie’s (1996) method of selecting uncommon names achieves the second lowest Type I error rate at 25 percent. These error rates are consistent with Massey (2017) who uses contemporary administrative data linked by Social Security Number as the ground truth. She finds that methods similar to Ferrie (1996) are associated with 19 to 23 percent Type I error rates. Abramitzky, Boustan, and Eriksson (2014)'s refinement of Ferrie (1996) increases match rates to 40 percent, but only half of the added links appear to be true links, as the Type I error rate increases to 32 percent. Feigenbaum’s (2016) supervised, regression-based machine learning model produces a Type I error rate of 34 percent when using the Iowa coefficients, and the Type I error rate decreases to 29 percent when estimated using LIFE-M data. Finally, Abramitzky, Mill, and Pérez (2018)’s less conservative cut-off results in the highest error rate at 37 percent. In terms of missed links, Ferrie (1996) correctly linked the lowest share of the sample without error, and other methods correctly linked 24 to 29 percent. Feigenbaum (2016)’s algorithm correctly linked 34 
percent of the LIFE-M sample with the coefficients from the Iowa census and 37 percent of the sample using the LIFE-M data, yielding the lowest rate of missed links (Type II errors). Importantly, Feigenbaum (2016) allows researchers to place different weights on Type I and Type II errors, even though Feigenbaum's implementation weights Type I and Type II errors equally. Similarly, researchers could adjust the parameters in Abramitzky, Mill, and Pérez (2018) algorithm to place different weights on Type I and Type II errors. Our Appendix Figures A1-A7 shows how show altering the penalty for Type I and Type II errors impacts results in both cases.

Next, Table 2 describes the representativeness of the linked sample. Because birth certificates do not contain socio-demographic measures found in the Census (race, age, or incomes of the parents), we regress a binary dependent variable (1= linked records) on a variety of individual covariates from the birth certificates. These variables include the individual's exact date of birth; ${ }^{23}$ the number of siblings in the family; the number of characters in the infants' (boys'), mothers', and fathers' names—a characteristic which is strongly positively correlated with years of schooling and income from wages in the 1940 Census; and the share of family records with a misspelled mother's or father's name, which we expect to be negatively correlated with years of schooling and income (Aizer et al. 2016). ${ }^{24}$ Table 2 presents the Waldstatistic for tests of whether these covariates are jointly associated with an observation being linked (pvalue beneath). If a representative set of birth certificates were linked, then these characteristics would not be jointly related to whether an observation was linked. However, Wald-statistics for the joint test of the association of these characteristics with linking show a persistent association. For all methods, including LIFE-M’s clerical review, we reject representativeness at the 1-percent level.

The signs and magnitudes of the regression results provide clues about the individuals easier to link (see the full set of regression results in Appendix C). Many automated methods are more likely to link boys

\footnotetext{
${ }^{23}$ Exact day of birth (1-366, due to leap years) is as close to a continuous measure as we can get in historical records, and season of birth is strongly correlated with socio-economic characteristics in modern data (Buckles and Hungerman 2013).

${ }^{24}$ We measure misspellings in father and mothers' names as the number of name spellings in the birth certificates of all siblings that differ from the modal spelling divided by the total number of children in a family.
} 
with higher incidence of misspelled father's last name, but more likely to link boys with a longer mother's name. All methods except Feigenbaum (2016) with estimated coefficients are more likely to link children with longer names, indicating that these linked records may come from more affluent families. At the same time, some methods are more likely to link individuals with more siblings, while other methods are more likely to link individuals with fewer siblings. In short, even though no linking algorithm appears to generate representative samples, different algorithms yield samples that are non-representative along different characteristics.

Our last analysis in Table 3 tests for the systematic correlation of links rejected in hand review with birth certificate characteristics using the same method. The only change from what is presented in Table 2 is that in these regressions the binary variable is equal to 1 if the observation's link was rejected by data trainers in a blind review. If the rejected links are systematically related to baseline characteristics, one might worry that the algorithm introduces systematic measurement error in variables of interest. Column 1 of Table 3 reports the heteroskedasticity-robust Wald-statistic (p-value beneath) by method for the LIFEM data (see the full set of regression results are in Appendix D). Again, for each algorithm, we reject the null hypothesis that errors in linking are unrelated to baseline characteristics at the 1-percent level. False links are significantly negatively associated with the length of a mother's name and length of a father's name in nearly all samples, suggesting that being falsely linked is negatively associated with affluence. Patterns across other variables are more varied. For example, the number of siblings is positively associated with the probability that a link is incorrect for the Feigenbaum (2016) algorithm, but the number of siblings is negatively associated with the probability that a link is incorrect in all of the Abramitzky, Boustan, and Eriksson (2014) samples. In short, different algorithms may induce different types of systematic error.

\section{B. Evaluating Algorithms Using the Synthetic Ground Truth and Early Indicators Samples}

One critique of these findings is that LIFE-M trainer decisions may be incorrect, even after the blind review process. This could lead the levels of Type I errors in the LIFE-M analysis to be too high or too low relative to the truth. To address this potential issue, we reevaluate algorithm performance in 
synthetic data (where the truth is known). Because this objective truth is not influenced by human reviewers at all, this validation exercise validates the results obtained from the LIFE-M project's human review. We also evaluate the same algorithms using the Early Indicators, data, proving a complimentary perspective using a sample that was linked by genealogists and is known to be highly accurate.

For both the synthetic and Early Indicators data, Table 1 compares the match rates and error rates for each prominent algorithm. Recall, for the synthetic data, a perfect match rate is 85 percent, because 15 percent of the original links are absent by design. Patterns in match rates across methods are slightly higher in the synthetic data but generally within a few percentage points of the LIFE-M match rates, with the exception of Feigenbaum (2016) and Abramitzky, Mill, and Pérez (2018). Notably, both methods perform substantially better in the synthetic data than in the LIFE-M data with a match rate of 56 and 57 percent in Feigenbaum (2016) with the Iowa and estimated coefficients, and 52 and 32 percent for Abramitzky, Mill, and Pérez (2018) in the less and more conservative versions, respectively. The match rates for Early Indicators' veterans linked to the 1900 complete count Census are generally higher than in the LIFE-M sample. This pattern is due at least in part to the fact that we are linking a sample of individuals that are known to be linkable (i.e., did not die before 1900) and has been successfully linked by other methods. In contrast, we did not restrict the sample of LIFE-M birth certificates to those that were identified as links by the data trainers.

Table 1 also shows that patterns of error rates in the synthetic and Early Indicators data are similar to those in LIFE-M, with the best performing algorithms in LIFE-M continuing to be the best performing in the synthetic data and Early Indicators data. Figure 4 describes patterns of error rates graphically across algorithms and datasets. In most cases, the error rates are slightly lower in the synthetic data and Early Indicators data relative to the LIFE-M records, ranging from 11 to 33 percent. Because there was no hand linking involved in producing the synthetic data, similar error levels suggest that the results of the LIFE-M hand-linking process and blind review reflect true errors in the automated linking algorithms. Larger reductions in error rates for machine learning algorithms like Feigenbaum (2016) and Abramitzky, Mill, and Pérez (2018) suggest that these methods may be effective at detecting the simple errors we simulated. 
Lower error rates are expected in the Early Indicators data, because the Early Indicators data only contain individuals who have been successfully linked by genealogists. Consequently, Type I error rates in the Early Indicators data range from 10 to 24 percent versus 15 to 37 percent in the LIFE-M data. The fact that the patterns of error rates are the similar in all datasets, however, provides strong support for the notion that prominent machine methods in current practice make considerable errors.

The findings for representativeness in the synthetic and Early Indicators are also similar to the LIFE-M data: linked samples in the synthetic and Early Indicators data appear unrepresentative. Table 2 presents a heteroskedasticity-robust Wald-statistic from regressions of whether a record was linked on baseline covariates (described in the table notes; see full regression results in Appendix C) for both datasets. For the synthetic data, this exercise allows a particularly strong test of the hypothesis that the nonrepresentativeness of linked samples reflects the linking algorithm per se. Because we randomly dropped 15 percent of individuals, non-random attrition due to differential death, enumeration, or emigration is ruled out by construction. The only reason that the linked synthetic sample would not be representative is that the methods link certain groups more systematically than others. Consistent with the linking algorithms creating non-representative samples, the Wald-statistics and p-values in column 2 reject representativeness for all methods in the synthetic data at the 1-percent level. Most methods are less likely to link individuals with more siblings.

The Early Indicators data require that we use a slightly different set of covariates for this analysis. ${ }^{25}$ As in the LIFE-M and synthetic data, we reject that the linked samples are representative at the 1-percent significance level for all methods except for Abramitzky, Boustan, and Eriksson (2014). Nearly all methods are more likely to link individuals with U.S.-born mothers; some methods are more likely to link individuals with longer first or last names, while others exhibit the reverse correlation.

In a final analysis, Table 3 shows that false links appear to be systematically related to baseline

\footnotetext{
${ }^{25}$ For the synthetic dataset, we use the same covariates as in the LIFE-M data when considering representativeness. For the Early Indicators data, we use continuous variables in age and length of first and last names and dummy variables for speaks English, owns a farm, currently married, foreign born, day of birth by year, literacy, and foreign born status of parents.
} 
sample characteristics in both the synthetic and Early Indicators data. For all methods, we reject the null hypothesis that false links are unrelated to baseline covariates. In both the synthetic and Early Indicators datasets, these results suggest that false links are not random with respect to sample characteristics, a pattern that may complicate inference by introducing systematic measurement error into analyses. See Appendix $\mathrm{D}$ for the full set of regression results.

\section{Summary of Findings}

Prominent algorithms yield widely varying results—even using the same data and linking variables. In the LIFE-M data, match rates range from 28 to 52 percent, while the share of links rejected in the police line-up ranges from 15 to 37 percent and consequently the associated Type II error rate ranges from 63 to 79 percent. Notably, some methods achieve lower Type II error levels and Type I error levels than other methods, such as Feigenbaum (2016) with estimated coefficients compared to Abramitzky, Boustan, and Eriksson (2014). The share of links rejected by humans in the Early Indicators data is slightly lower and the match rates are higher, owing to the fact that we are only using a sample of individuals who have been linked by genealogists and may, therefore, be more easily linked than other records. A synthetic ground truth dataset confirms these patterns and also suggests that machine learning algorithms like Feigenbaum (2016) and Abramitzky, Mill, and Pérez (2018) are effective at detecting and correcting for synthetic errors, which speaks to their potential value in improving the quality of linked data.

An equally important finding is that linking error rates vary across datasets—even when links are created using the same algorithm and linking variables. This variation across datasets cautions against generalizing too broadly from this paper’s findings, although Type I and Type II error rates may be high in other datasets as well. These findings recommend that researchers examine their linked samples for clues about error rates, representativeness, and systematic measurement error. But they also beg the question: why do error rates vary so much across algorithms—-the question we consider next.

\section{How Variations in Algorithms Alter Method Performance}

Understanding what drives differences in algorithm performance is key to improving existing 
methods and current practice. This section considers how the performance of these algorithms changes when varying key features of their set-ups. First, we examine the role of different phonetic name cleaning strategies for algorithms that require agreement in cleaned names, specifically Ferrie (1996) and Abramitzky, Boustan, and Eriksson (2014). Second, we extend the Ferrie (1996) algorithm to include more common names. Third, we examine the consequences of equal weighting of exact ties (i.e., multiple, exact matches). A final section examines the robustness of these findings across all methods to using middle names, information on race, and extensions to population-to-population linking.

\section{A. Phonetic Name Cleaning, Common Names, and Ties}

Phonetic string cleaning algorithms are designed to account for orthographic differences that could lead a true match to be missed, such as minor spelling differences, name Anglicization, and transcription/digitization errors. Figure 3 and Table 4 show how the performance of the Ferrie (1996) and Abramitzky, Boustan, and Eriksson (2014) algorithms vary with three types of different phonetic name cleaning: no cleaning (labeled "Name”), Soundex (labeled “SDX”), and NYSIIS. Interestingly, although name cleaning is intended to increase match rates, it can also decrease match rates if it increases ties (by removing meaningful spelling variations). This interaction is important for the Ferrie (1996) method, which matches between 20 and 33 percent of baseline sample depending on the phonetic name cleaning used. This method's uncommon name restriction (i.e., allowing fewer than 10 potential matches in the 1940 Census regardless of age) results in the match rate falling from 33 (Name) to 28 (NYSIIS), to 20 percent (Soundex), because this cleaning creates more common name strings, leading the algorithm to discard more links due to ties. Abramitzky, Boustan, and Eriksson (2014) does not show such dramatic reductions, with match rates ranging from 39 to 42 percent.

As shown in Table 4 and Figure 3, the likelihood of Type I errors increases with the use of phonetic name cleaning in Ferrie (1996) and Abramitzky, Boustan, and Eriksson (2014). Using NYSIIS rather than uncleaned names increases Type I error rates by an average of 18 percent, ranging from as little as 5 to as much as 25 percent across datasets. Using Soundex rather than uncleaned names increases Type I error 
rates by an average of 36 percent, ranging from 14 to as much as 64 percent. These increases occur because, in addition to orthographic and transcription errors, phonetic codes remove meaningful spelling variation from names. For example, both Soundex and NYSIIS would code "Meyer" and "Moore” as the same name, whereas reviewers tend to treat these as different names. Ironically, this dramatic increase in error rates induced by the use of phonetic name cleaning universally decreases the share of the sample that is correctly linked.

Another modification to Ferrie (1996) is to link more common names. Recall that-for computational reasons-Ferrie (1996) discarded matches if there were 10 or more candidate matches, regardless of age differences. We relax this assumption and include records with 10 or more candidates when we find links (labeled “Ferrie 1996 + common names”). Table 4 and Figure 3 show that including common names results in significantly higher match rates, including higher shares of true links than in the original method. Interestingly, the results are almost identical to those of Abramitzky, Boustan, and Eriksson (2014), which is because this method's key deviation from Ferrie's (1996) is attempting to link more common names. The inclusion of common names roughly doubles the share of the LIFE-M sample that is incorrectly linked, but it also decreases Type II error rates.

Similar in spirit to the common names restriction, Abramitzky, Boustan, and Eriksson (2014) implement a robustness check that discards links if a name tie occurs within a two-year age radius. As reported in Figure 4 under Abramitzky, Boustan, and Eriksson 2014 (NYSIIS, Robustness), this restriction lowers the match rate to 24 percent but it also halves the share of the sample that is incorrectly linked, from 14 percent to 6 percent, and changes the Type I error rate from 32 percent to 23 percent. Because this restriction is very similar to the uncommon names restriction, this algorithm performs almost identically to Ferrie (NYSIIS) in terms of match rates and Type I errors. Relatedly, the Abramitzky, Mill, and Pérez (2018) algorithm with more conservative cut-offs is also similar to an uncommon names restriction. By requiring a high threshold of the probability of a candidate match being a correct match, and requiring the second-best options for the observations in the match to be much lower, this restriction ensures that the links made used under this restriction have no close analogues either due to differences in spelling or age. 
This unsupervised approach slightly outperforms Ferrie (NYSIIS) in terms of Type I and Type II error rates.

A third variation on prominent algorithms relates to how ties are handled (e.g., cases like M3 in Figure 1C and M4 in Figure 1D). The incidence of ties in contexts with limited information (such as matching between U.S. Censuses) is very high and has important consequences for linking. If one could break exact ties or use ties in the analysis, researchers could match the majority of the sample, raising match rates substantially. For the Ferrie (1996) algorithm, using both common names and ties raises the match rates from 20 to 33 percent to 69 to 86 percent.

Two main approaches to using exact ties have been suggested by the literature. First, the statistics literature offers an alternative to tie-breaking by using probability weighting. For instance, one could use a weight that is the conditional probability that the match is correct (Scheuren and Winkler 1993, Lahiri and Larsen 2005). In the absence of other data features, this suggestion simplifies to weighting by $1 / J_{r}$, where $J_{r}$ is the number of exact ties for record $r{ }^{26} \mathrm{Nix}$ and Qian (2015)'s random selection among ties is similar in spirit. Their process draws one of the candidate matches with probability $1 / J_{r}$. Importantly, simple probability weighting and random selection among ties have the same expected performance in certain contexts such as those we consider later for our case study. ${ }^{27}$ We label results that include ties as "Ferrie 1996 + ties.” Table 4 shows that including ties may dramatically increase match rates, but Figure 4 shows that this substantially increases the share of observations that are incorrectly matched in every sample. This makes sense, because at most one of the candidate links can be correct. For instance, if there are ten candidate "John Smith" links and only one of these is the correct link, nine out of ten of these links are incorrect. Notably, the Type I error rate increases slightly more in the LIFE-M and synthetic data than in

\footnotetext{
${ }^{26}$ This simple probability weighting differs from Lahiri and Larsen (2005), because match probabilities vary in their data due to a specifically defined data generating process, and they are able to trim candidate links with lower match probabilities. We do not assume a specific data generating process. Furthermore trimming is not possible when all records are equally tied.

${ }^{27}$ We explain this result later in more detail, but the intuition is straightforward. Let $N=$ the number of observations, $M=$ the number of primary records with multiple exact ties as their best matches, $J_{r}$ the number of ties for a primary record $r=1,2, \ldots, M$. Assuming that one of the ties is the correct link, the expected number of false matches for records with ties is $\sum_{r=1}^{M}\left(J_{r}-1\right) / J_{r}=M-$ $\sum_{r=1}^{M} 1 / J_{r}$ for both random selection and simple probability weighting. As the number of multiples increases for a given record, the probability weight on a false match gets smaller as does the weight on the true match. The results from probability weighting may differ slightly due to sampling variation.
} 
the Early Indicators data, which may reflect the fact that the Early Indicators data are a selected group of observations that genealogists successfully linked, and may have fewer close ties.

Figure 5 describes the mixed progress in historical automated linking since 1996. As the literature has moved from the use of Ferrie's (1996) uncommon name sample and increased match rates, some methods have also increased Type I errors (and decreased precision). The hope of researchers using these methods is that—on net—-they are increasing the share of true links in their sample as well as sample representativeness. However, for the synthetic and Early Indicators data, the pattern of Type I and Type II errors suggest that there may be scope to improve in both dimensions by leveraging the strengths of different algorithms. $^{28}$

Finally, we find that these variations and robustness checks do not tend to make samples or false links more representative. Table 5 shows that the data can reject representativeness at standard levels for almost all algorithms and datasets. Similarly, Table 6 shows that variations in algorithms produce false links that are systematically related to baseline sample characteristics in all datasets.

\section{B. Robustness: Middle Names, Race, and Population-to-Population Linking}

How much should we expect these results to change with the addition of information commonly available in historical datasets? A first robustness check considers how the addition of middle name or race could reduce Type I error rates. For middle names, we examine a subsample of cases where middle name or initial was available for both the birth certificate and the linked Census record. Then, we calculate the number of false links that would have been eliminated had the automated method required that middle initial match for all potential matches after running a matching algorithm. We apply this restriction ex post, but it would also be possible to include middle name agreement in the matching process as a feature considered by an algorithm in the process of making matches.

\footnotetext{
${ }^{28}$ Of course, the level of Type II errors in the LIFE-M and synthetic data is overstated, because some infants did not survive until the 1940 Census, emigrated, or were missed by enumerators in the 1940 Census. We estimate that these factors likely account for around 15 percent of missed links (see footnote 16). A linking method that linked all LIFE-M or synthetic data individuals correctly would locate at $(0.15,0)$, missing only the 15 percent individuals who are unlinkable and making no errors. Because these sources of attrition affect all methods equally, these factors do not influence our comparisons across methods.
} 
Table 7 shows that the availability of middle initials may reduce match rates but also the reduce rate of false matches. Columns 1 and 2 reprint the information on match rates and Type I error rates from Table 4. Column 3 shows the share of matched observations that have information on middle initial in the birth certificate and the Census record, which range from a quarter to a third of matches. Column 4 reports the share of matches that have discordant middle initials among the subset of matches that have middle names in both records, ranging from 20 percent to 57 percent. Column 5 reports the Type I error rate among the matches with discordant middle initials. What is clear is that the Type I error rate is always above 87 percent within this subset. Presumably, these error rates are high because disagreements among middle initials mattered to the trainers considering these observations when making matches. Finally, columns 6 and 7 recalculate the match rate and type I error rates after dropping observations that have discordant middle initials. Match rates tend to drop by several percentage points, but since these observations dropped had a high error rate, the Type I error rates drop by more. For example, Ferrie (1996) with NYSIIS drops from a match rate of 28 percent and an associated Type I error rate of 25 percent to a match rate of 30 percent and an associated Type I error rate of 15 percent. Although this change does not affect the ranking of prominent algorithms in terms of Type I error rates, using middle initials when available reduces Type I error rates across the board. The Type II error rates are nearly unchanged despite the drop in match rates, with all changes in Type II error rates never exceeding one percentage point. This result suggests that the addition of more information contained in middle names can substantially reduce Type I linking errors with minimal changes in Type II errors, at least among observations that have middle initials in the LIFE-M data.

A second robustness check compares race indicated on the 1940 Census for LIFE-M linked records to race indicated on the 1940 Census for other links. ${ }^{29}$ Note that race is only observable for the observations that LIFE-M successfully linked, as we infer race from the 1940 Census. Column 3 of Table 8 shows the

\footnotetext{
${ }^{29}$ To the extent that some individuals "pass" for other races, this robustness check may eliminate true links (Nix and Qian 2015, Mill 2013).
} 
share of linked birth certificates that would not have been erroneously linked by an algorithm that blocked on race. Interestingly, only a small share of incorrect links have discordant races, ranging from 0 to 5 percent. When we omit incorrect links with discordant races in column 5, we find that the match rate only drops slightly and the race-adjusted Type I error rates decrease by no more than two percentage points across methods. This is consistent with Massey (2017), who finds that linking errors in linking the 2005 Current Population Survey to the Numident only decreased by 0.07 percentage points when blocking on race. In contrast to using middle initial, race does not appear to add much information (beyond what is contained in first and last name) that impacts the share of correct matches. Note, however, that including race may result in better link disambiguation among links that appear to be close substitutes, and our test does not illuminate how effective race might be if used in this way.

A third robustness check examines to the implications of linking a sample (rather than a population) to a Census. The critical difference between these two settings is that an observation could appear to be unique in a sample while having duplicates or near-duplicates in the population. This is important because many algorithms drop a match if (1) it occurs more than once in the set of records to be linked or (2) more than one observation links to the same record. Both are more likely to occur for a population than a sample. Sample-to-population linkage may, therefore, result in a higher share of incorrect matches than populationto-population linkage.

To quantify the importance of linking a sample to a population as we do here, we compare our results to matching the universe (e.g., the population) of birth certificates to the 1940 Census. First, we match the universe of Ohio and North Carolina birth certificates to the 1940 Census using each automated method, including adjustments described above. Then, we isolate attention to the subset of records in the LIFE-M sample to assess performance. Since the LIFE-M sample is a random subsample of birth certificates, we expect the results to generalize to the population.

Figure 6 displays the results, with the horizontal axis depicting Type I error rates in matching between the sample and the 1940 Census and the vertical axis displaying the same results matching the population of birth certificates and the 1940 Census. Results along the 45-degree line indicate perfect 
agreement in the two rates. As expected, false link rates fall below the 45-degree line for all methods that use the post-linking adjustments, suggesting that Type I error rates are somewhat higher for sample-topopulation linkage. The Type I error rate in Ferrie (1996) with exact names is 20 percent for a sample but 17 percent for the population; for Abramitzky, Boustan, and Eriksson (2014) with exact names the Type I error rate is 25 percent for the sample versus 20 percent for the population. However, the overall error rate remains high, with no method achieving an error rate lower than 15 percent in the population.

\section{Summary of Findings}

Variations on machine-linking algorithms may improve or worsen performance. Deterministic algorithms that clean names using Soundex or NYSIIS perform worse than using raw name strings. Similarly, trying to link common names (especially in conjunction with phonetic name algorithms) tends to increase error rates and also tends to increase the share of the initial sample correctly linked. Finally, tie breaking or weighting ties equally could dramatically increase sample sizes but may have the unintended effect of using more incorrect matches in analyses. Including middle names as a linking criterion appears to have large effects on Type I error rates. However, using race information or using population-topopulation linking appears to alter our results only modestly.

\section{HoW AutOMATED METHODS AFFECT INFERENCES}

Our final analysis explores the consequences of Type I and Type II errors for inferences about historical rates of intergenerational mobility. Following the intergenerational literature (Solon 1999, Black and Devereux 2011), we consider the following benchmark specification,

$$
\log (\mathrm{y})=\pi \log (x)+\varepsilon,
$$

where the dependent and independent variables have been rescaled to capture only individual deviations from population means. The dependent variable, $\log (y)$, refers to the log of son's wage income in adulthood in the 1940 Census. The key independent variable, $\log (x)$, refers to the parent's log wage income in the 1940 Census. Within this framework, we interpret $\pi$ as the intergenerational income elasticity. The magnitude of $\pi$ is an important indicator of the role that parents' income plays in determining their 
children's wage earnings. Intergenerational mobility is measured as $1-\pi$, which is often regarded as a metric of economic opportunity.

Our analysis uses the LIFE-M sample of 19,486 boys (43 percent of the 45,442 that were linked to the 1940 Census) and samples linked using different automated methods to estimate intergenerational mobility. Unlike other analyses using the Census and Panel Survey of Income Dynamics, we must link fathers from birth certificates to the 1940 Census to obtain their income information. Links for fathers are obtained using only the LIFE-M clerical review method, so that father links remain constant in all regressions. By using the same links for fathers and different methods to link sons, our analysis describes differences in the estimates that are driven by differences in methods used to link sons.

\section{A. How Type I Errors Affect Inferences}

Different kinds of Type I errors in links for sons may have vastly different implications for inferences about intergenerational mobility. Within the regression framework in equation (1), measurement error in son's income (the dependent variable in the regression) that is uncorrelated with father's income will still allow us to estimate $\pi$ consistently using OLS, though the estimates will be less precise. However, measurement error on the right-hand side in father's income (the independent variable in the regression) is more consequential. At first glance, considering measurement error in father's income seems counter to our problem of using different linking methods to link sons. Note, however, that linking a boy to the wrong man in 1940 is equivalent to assigning the wrong father's income to that man.

Our conceptual framework for thinking about linking-induced measurement error is similar to Horowitz and Manski (1995). We assume that a linking method, $\ell$, induces Type I error in matches by erroneously linking a father to a son (we do not derive bounds here, but that is a useful avenue for future research). The presence of this measurement error allows us to divide the sample into two groups, $g$ : one for which the links are correct, denoted with a *, and another for which the link is imputed (or incorrectly classified), i. Following Greene (2008) and Stephens and Unayama (forthcoming), we decompose the OLS estimate of $\pi$ for a sample linked with method, $\ell$, into the sum of within and between covariance for the 
correct, *, and imputed groups, $i . b$ denotes the between component. Let $s_{x y}^{\ell *}+s_{x y}^{\ell \mathrm{i}}=\sum_{\mathrm{g}} s_{\mathrm{xy}}^{\ell \mathrm{g}}=$ $\left.\sum_{\mathrm{g}} \sum_{\mathrm{k}}\left(\log \left(\mathrm{x}_{\mathrm{kg}}\right)-\overline{\log \left(\mathrm{x}_{\mathrm{kg}}\right.}\right)\right)\left(\log \left(\mathrm{y}_{\mathrm{kg}}\right)-\overline{\log \left(\mathrm{y}_{\mathrm{kg}}\right)}\right), \quad \mathrm{s}_{\mathrm{xy}}^{\ell \mathrm{b}}=\sum_{\mathrm{g}} \mathrm{N}_{\mathrm{g}}\left(\overline{\overline{\log \left(\mathrm{x}_{\mathrm{kg}}\right)}}-\overline{\overline{\log \left(\mathrm{x}_{\mathrm{kg}}\right)}} \overline{\overline{\log \left(\mathrm{y}_{\mathrm{kg}}\right)}}-\right.$ $\left.\left.\overline{\overline{\log \left(\bar{y}_{\mathrm{kg}}\right.}}\right)\right)$ where group means are defined with a single bar and overall means are defined by two bars, such that,

$$
\widehat{\pi}^{\ell}=\frac{s_{\mathrm{xy}}^{\ell}}{s_{\mathrm{xx}}^{\ell}}=\frac{s_{\mathrm{xy}}^{\ell *}+s_{\mathrm{xy}}^{\ell \mathrm{i}}+s_{\mathrm{xy}}^{\ell \mathrm{b}}}{s_{\mathrm{xx}}^{\ell}}=\frac{s_{\mathrm{xx}}^{*}}{s_{\mathrm{xx}}} \widehat{\pi}^{\ell *}+\frac{s_{\mathrm{xx}}^{\mathrm{i}}}{s_{\mathrm{xx}}} \widehat{\pi}^{\ell \mathrm{i}}+\frac{s_{\mathrm{xx}}^{\mathrm{b}}}{s_{\mathrm{xx}}} \widehat{\pi}^{\ell \mathrm{b}} .
$$

Equation (2) shows that an OLS estimator converges in probability to a weighted average of the plim for the correct links, $\widehat{\pi}^{\ell *}$, imputed links, $\widehat{\pi}^{\ell \mathrm{i}}$ and the between group term (* versus $\left.i\right)$, $\widehat{\pi}^{\ell \mathrm{b}}$, where the weights on each term reflect the share of variance due to each component, $\theta$ :

$$
\operatorname{plim} \widehat{\pi}^{\ell}=\theta^{\ell *} \operatorname{plim} \widehat{\pi}^{\ell *}+\theta^{\ell \mathrm{i}} \operatorname{plim} \widehat{\pi}^{\ell \mathrm{i}}+\theta^{\ell \mathrm{b}} \operatorname{plim} \widehat{\pi}^{\ell \mathrm{b}} .
$$

The between group component can be thought of as the "selection" term. In some cases, we expect that the plim of the between term to be zero (e.g., if the means of son's income or father's income are the same for the imputed and correctly linked groups). This pattern could happen in practice if errors (e.g., enumeration or transcription error) randomly assign records to these groups. Initially, we assume this term is zero to simplify exposition but later relax this assumption. Furthermore, note that if the variances of father income are equal across all groups, the weights $\theta$ become the share of the sample in each category.

Now, consider the probability limit of the two remaining non-weight terms, $\widehat{\pi}^{\ell *}$ and $\widehat{\pi}^{\ell \mathrm{i}}$. The first term represents the elasticity for the linked subsample, plim $\widehat{\pi}^{\ell *}=\pi$. The second term is an estimated elasticity for the imputed observations. If we assume $\operatorname{cov}\left(\varepsilon, \log \left(\mathrm{x}^{\ell \mathrm{i}}\right)\right)=0$, then

$$
\operatorname{plim} \widehat{\pi}^{\ell \mathrm{i}}=\frac{\operatorname{cov}\left(\log \left(\mathrm{y}^{*}\right), \log \left(\mathrm{x}^{\ell \mathrm{i}}\right)\right)}{\operatorname{var}\left(\log \left(\mathrm{x}^{\ell \mathrm{i}}\right)\right)}=\frac{\operatorname{cov}\left(\pi \log \left(\mathrm{x}^{*}\right)+\varepsilon, \log \left(\mathrm{x}^{\ell \mathrm{i}}\right)\right)}{\operatorname{var}\left(\log \left(\mathrm{x}^{\ell \mathrm{i}}\right)\right)}=\pi \frac{\operatorname{cov}\left(\log \left(\mathrm{x}^{*}\right), \log \left(\mathrm{x}^{\ell \mathrm{i}}\right)\right)}{\operatorname{var}\left(\log \left(\mathrm{x}^{\ell \mathrm{i}}\right)\right)}
$$

If the imputed father's income is the same as the true father's income, $\log \left(\mathrm{x}^{*}\right)=\log \left(\mathrm{x}^{\ell \mathrm{i}}\right)$, then plim $\widehat{\pi}^{\ell *}=$ $\operatorname{plim} \widehat{\pi}^{\mathrm{i}}$. However, if $\frac{\operatorname{cov}\left(\log \left(\mathrm{x}^{*}\right) \log \left(\mathrm{x}^{\ell \mathrm{i}}\right)\right)}{\operatorname{var}\left(\log \left(\mathrm{x}^{\ell \mathrm{i}}\right)\right)} \neq 1$, then plim $\widehat{\pi}^{\ell \mathrm{i}} \neq \pi$ and the degree of the inconsistency depends on the relationship between the true and imputed father's income.

There are several special cases of interest. First, suppose that there is no relationship between the true father's income and the imputed father log income, or that $\frac{\operatorname{cov}\left(\log \left(x^{*}\right), \log \left(x^{\ell \mathrm{i}}\right)\right)}{\operatorname{var}\left(\log \left(\mathrm{x}^{\ell \mathrm{i}}\right)\right)}=0$. Then, the plim $\widehat{\pi}^{\ell \mathrm{i}}=$ 0 and the estimator is inconsistent in proportion to the share of imputed links, plim $\widehat{\pi}^{\ell}=\theta^{\ell *} \pi$. Second, 
consider the case where imputed father's income equals the true father's income plus noise, or $\log \left(\mathrm{x}^{\ell \mathrm{i}}\right)=$ $\log \left(\mathrm{x}^{*}\right)+\mathrm{u}$. Under the assumptions of the classical errors in variables model $(\mathrm{plim}(\mathrm{u} \varepsilon)=0$, $\operatorname{plim}\left(\operatorname{ulog}\left(x^{*}\right)\right)=0$, and $\left.\operatorname{plim}(\operatorname{ulog}(y))=0\right)$, then plim $\widehat{\pi}^{\ell \mathrm{i}}=\theta^{\ell \mathrm{i}}\left(\frac{\sigma_{\mathrm{x}}^{2}}{\sigma_{\mathrm{x}}^{2}+\sigma_{\mathrm{u}}^{2}}\right) \pi$. Moreover, plim $\widehat{\pi}^{\ell}=$ $\left(1-\theta^{\ell *}\right) \pi+\theta^{\ell *}\left(\frac{\sigma_{\mathrm{x}}^{2}}{\sigma_{\mathrm{x}}^{2}+\sigma_{\mathrm{u}}^{2}}\right) \pi$. Third, it is well known that non-classical measurement error for the imputed fathers could lead to under or over-statement of the parameter of interest, plim $\widehat{\pi}^{\ell \mathrm{i}}>\pi$ or plim $\widehat{\pi}^{\ell \mathrm{i}}<\pi$.

As a final exercise, consider the effect of Type I errors on inference using exact ties. Consider a setting where $N$ is the total number of records that one wishes to link and for $M \leq N$ of these records, $r=1,2$, $\ldots, M$, there are $J_{r}$ candidate matches that are tied. For instance, if the first record with ties involves 30 potential matches for a John Smith, age 40, then for $r=1, J_{1}=30$. A second record, however, may only have 4 ties, so $r=2$ and $J_{2}=4$. Assume that there is one correct link among the ties for record, $r$, indexed by $j=1$, $\log (y)$, and imputed (but incorrect links) $\log \left(\mathrm{y}_{j}\right), j=2, \ldots, J_{\mathrm{r}}$. From the researcher's perspective, the correct link is unknown and the probability that any one of the ties is correct is $\frac{1}{\mathrm{~J}_{\mathrm{r}}}$.

Assume that one of these records would be selected at random to use in the analysis. By the same logic as in equation (2), a regression estimate of the intergenerational income elasticity can be decomposed into a variance-weighted sum of elasticities for three groups of observations - correct, unique links, denoted *; a correct link from the ties, denoted $j=1$; incorrect links from the ties, denoted $j>1$, and a "selection term" (which we assume is zero). Therefore, the estimated elasticity will be $\widehat{\pi}^{\ell}=\frac{s_{x x}^{*}}{s_{x x}} \widehat{\pi}^{* \ell}+\frac{s_{x x}^{j=1}}{s_{x x}} \widehat{\pi}^{j=1, \ell}+$ $\frac{\mathrm{s}_{\mathrm{xx}}^{\mathrm{j}>1, \ell}}{\mathrm{s}_{\mathrm{xx}}}\left(\sum_{\mathrm{j}=2}^{\mathrm{J}_{\mathrm{r}}} \frac{\mathrm{s}_{\mathrm{xx}}^{\mathrm{j}, \ell}}{\mathrm{s}_{\mathrm{xx}}^{\mathrm{j}>1, \ell}} \widehat{\pi}^{\mathrm{j} \ell}\right)$ and $\operatorname{plim}\left(\sum_{\mathrm{j}=2}^{\mathrm{J}_{\mathrm{r}}} \frac{\frac{\mathrm{j}}{\mathrm{xx}}_{\mathrm{x}_{\mathrm{xx}}^{\mathrm{j}}}^{\mathrm{j}>1, \ell}}{\pi^{\mathrm{j}} \ell}\right)=\sum_{\mathrm{j}=2}^{\mathrm{J}_{\mathrm{r}}} \lambda_{\mathrm{j}} \operatorname{plim} \pi^{\mathrm{j} \ell}$. Therefore, the estimate of the intergenerational income elasticity using random selection to break ties is,

$$
\operatorname{plim} \widehat{\pi}^{\ell}=\pi\left[\theta^{* \ell}+\theta^{\mathrm{j}=1, \ell}+\theta^{\mathrm{j}>1, \ell}\left(\sum_{\mathrm{j}=2}^{\mathrm{J} r} \lambda_{\mathrm{j}} \Psi_{\mathrm{j} \ell}\right)\right]
$$

Note that this estimator is inconsistent if $\psi_{\mathrm{j} \ell}=\frac{\operatorname{cov}\left(\log \left(\mathrm{x}_{1}\right), \log \left(\mathrm{x}_{\mathrm{j}}\right)\right)}{\sigma_{\mathrm{x}_{\mathrm{j}}}^{2}}<1 .{ }^{30}$ The degree of inconsistency is, again, determined by how much information is in the incorrect ties. If the weights, $\theta$ and $\lambda$, simplify to the expected shares of observations in each group (as they would if variances were equal across all groups as we note above), the degree of inconsistency is also related to the share of all records with exact ties, $\frac{\mathrm{M}}{\mathrm{N}}$ (implicit in the weight), as well as the number of multiples for each record, $\mathrm{J}_{\mathrm{r}}$.

${ }^{30}$ Note that $\frac{\operatorname{cov}\left(\log \left(y_{j}\right), \log \left(x_{1}\right)\right)}{\sigma_{x_{j}}^{2}}=\frac{\operatorname{cov}\left(\pi \log \left(x_{j}\right)+\varepsilon, \log \left(x_{1}\right)\right)}{\sigma_{x_{j}}^{2}}=\frac{\operatorname{cov}\left(\log \left(x_{1}\right), \log \left(x_{j}\right)\right)}{\sigma_{x_{j}}^{2}} \pi=\operatorname{plim}\left(\pi^{j}\right)$. 
These conclusions are identical if the elasticity is estimated with a probability-weighted estimator where the weight is the probability that any exact multiple in a set of exact multiples is the true match, or $1 / J_{r}$. The probability limit of the estimator will be the same, although the performance of these methods may diverge in smaller samples. ${ }^{31}$ This result is intuitive because the same share of imputed observations would be present using probability weighting or random selection for exact ties. In summary, the presence of imputed links - either through random selection or probability weighting-will generally lead to inconsistency, with the degree of inconsistency increasing in the number of records with ties, the number of exact ties for a given record, as well as the relationship between imputed observation and the truth. After examining the role of Type II errors, we examine the quantitative importance of these errors in a case study.

\section{B. How Type II Errors Affect Inferences}

Social scientists are accustomed to working with small representative samples. As long as links are representative of the underlying population, higher Type II error rates should only reduce precision. Across linking methods and datasets, however, this paper finds evidence that samples of links are not representative. If Type II errors result in the selective representation of different groups and the relationship of interest is heterogeneous across these groups, Type II errors may also lead to inconsistent estimates of population parameters in linked samples.

Heterogeneity in intergenerational income elasticities is believed to exist for many reasons. For instance, researchers have concluded that intergenerational income elasticities are larger for blacks than whites (Duncan 1968, Margo 2016) and that patterns of mobility are substantially different for farmers compared to non-farmers (Hout and Guest 2013, Xie and Killewald 2013). If one group is over-represented in the linked data, this will bias inferences about the historical rate of the population's intergenerational mobility.

To make this point concretely, assume that the two groups in equation (3) are high mobility, $h$, and

\footnotetext{
${ }^{31}$ Reducing the influence of observations with less information is why some statisticians recommend truncating lower probability links, where presumably the covariance between the income of the father for the imputed link and the true link is small (Scheuren and Winkler 1993, Lahiri and Larsen 2005). Although Lahiri and Larsen (2005) propose an exactly unbiased estimator of $\pi$, this result only holds when the estimated link probability is uncorrelated with father's income and where an exact data generating process for links is estimated. But this result breaks down in many historical settings, because the distribution of matching variables (name, age, and birth place) are correlated with outcomes and, often, a parent's socioeconomic status (see Appendix C and D).
} 
low mobility, l (rather than correctly and imputed links). Denote the intergenerational income elasticities of these groups as $\pi^{\mathrm{h}}$ and $\pi^{\mathrm{l}}$ where (where $\pi^{\mathrm{h}} \leq \pi^{\mathrm{l}}$ ), and the share of the variation attributable to each group is $\theta^{\mathrm{h}}$ and $\theta^{\mathrm{l}}$, respectively. Finally, assume that there are no errors in linking. Therefore, following the logic of equation (2), the regression estimate of the population elasticity parameter for a given linking method, $\ell$, is,

$$
\operatorname{plim} \widehat{\pi}^{\ell}=\theta^{\ell \mathrm{h}} \text { plim } \widehat{\pi}^{\ell \mathrm{h}}+\theta^{\ell l} \operatorname{plim} \widehat{\pi}^{\ell l}+\theta^{\ell \mathrm{b}} \text { plim } \widehat{\pi}^{\ell \mathrm{b}}
$$

The inconsistency of the probability limit in equation (5) depends upon several factors. First, if $\pi^{\mathrm{h}}=\pi^{l}$ and the means for both groups of fathers and sons are the same, the selection term is 0 and having a nonrepresentative sample will not affect inference. Having a representative sample matters only to the extent that the relationship of interest varies across those groups or the group's characteristics differ. Second, if $\pi^{\mathrm{h}} \neq \pi^{l}$ (and the group means are the same), Type II errors that effectively decrease the share of variation attributable to one group will lead to an inconsistent estimate of the population intergenerational elasticity parameter. Suppose that a linking method introduces Type II errors, which effectively decreases the variation attributable to observations representing the low mobility group. (In the extreme, high rates of Type II errors could imply that none of the total variation is attributable to low mobility group.) These Type II errors would result in an elasticity estimate that puts lower weight on the low-mobility group, resulting in a lower estimated elasticity. Third, if $\pi^{\mathrm{h}}=\pi^{l}$ but the group means are different, then the selection term will not be 0 and inferences will be affected in an ambiguous way. Both heterogeneity and selection, of course, may vary greatly across samples. The following case study examines the combined implications of non-representativeness (through heterogeneity and selection) using inverse propensity weights to adjust for differences in observed characteristics (DiNardo, Fortin, and Lemieux 1996, Heckman et al. 1998).

\section{Results: Intergenerational Elasticity Estimates from the 1940 Census}

Different linking methods could have large effects on intergenerational income elasticity estimates through their influence on both Type I and Type II error rates. Figure 7A reports estimates of the intergenerational elasticity of income using samples of sons linked using different methods. For the LIFE- 
M links, we estimate an income elasticity of 0.24 between fathers and sons. Consistent with lifecycle bias and transitory income fluctuations attenuating our estimates, this estimate is lower than modern estimates. ${ }^{32}$ These biases, however, should not affect our comparisons across different linking methods for the same set of records.

Several important patterns emerge. First, higher Type I errors in matching tend to be associated with smaller intergenerational elasticities. Consistent with attenuation described in equations (4) and (5), estimates using linking samples with higher Type I error rates tend to be smaller. Using NYSIIS and Soundex tends to increase Type I error rates and produce smaller estimates than using the reported name. Moreover, weighting ties results in Type I error rates ranging from 50 to 67 percent and yields intergenerational income elasticity estimates of 0.19 to 0.11 . However, Type I error is not the only factor determining bias. It is notable that the more conservative Abramitzky, Mill, and Pérez (2018), the method with the lowest Type I error rates, yields an intergenerational income elasticity that is 20 percent smaller and statistically different than the true coefficient. Conversely, a method with a comparatively high Type I error rate such as Feigenbaum (2016) achieves an estimated intergenerational income elasticity that is statistically indistinguishable from the LIFE-M elasticity. These results may reflect the fact that sample composition or a more systematic correlations of the errors with certain characteristics impact the coefficient.

To examine the role of non-representativeness, we use inverse propensity-score weights to reweight the linked sample to have the characteristics of the LIFE-M birth certificate sample (Bailey, Cole, and Massey 2018). ${ }^{33}$ Figure 7B shows that the reweighted intergenerational income elasticities tend to be

\footnotetext{
${ }^{32}$ For instance, Chetty et al. (2014) estimates 0.33 , which is itself smaller than estimates for the same period using survey data (Mazumder 2015). Life-cycle bias may attenuate the estimated intergenerational elasticity regardless of matching method (Mazumder 2005, Haider and Solon 2006, Black and Devereux 2011, Mazumder 2015). In addition, wage income observed in the 1940 Census is an imperfect measure of permanent income, and we expect the single year observation of income for both generations can generate downward bias in estimated elasticities due to the importance of transitory income (Solon 1992, Zimmerman 1992, Mazumder 2005). On the other hand, the absence of farm and self-employed income in 1940 may lead this analysis to overstate mobility by excluding father-son pairs of farmers-an occupation that tends to be highly persistent across generations (Hout and Guest 2013, Xie and Killewald 2013). However, lifecycle bias and transitory income fluctuations should have similar effects for all methods.

${ }^{33}$ To construct these weights, we first run a probit model of link status (for each method) on covariates, $\boldsymbol{X}$, which include an
} 
slightly smaller in magnitude than the unweighted Figure 7A estimates. This result may stem from the modest over-representation of larger, less-mobile families in the linked sample. The attenuation of the coefficient for the more conservative Abramitzky, Mill, and Pérez (2018) is cut in half, however, by using weights. For this case study, however, the effects of non-representativeness (as measured by the changes induced by reweighting) on observed characteristics appear modest in comparison to the role of errors in linking. Of course, one might also choose to re-weight the sample to resemble the 1940 Census. Appendix E shows that these results are nearly identical to results presented here.

While estimates using machine-linked samples appear attenuated relative to LIFE-M, the attenuation is not always as severe as one might expect with random error. For instance, Ferrie (1996) with name results in a 20 percent Type I error rate but the intergenerational elasticity estimate obtained from these links is one percentage point different from the LIFE-M estimate. If the selection term in equation (3) were zero, and the signal to noise ratio in equation (4) were zero, one would expect to estimate 0.19 $(=0.80 * 0.24)$. Therefore, one might think that fathers' incomes for imputed links positively covaries with the truth or that the Ferrie (1996) is positively selected on immobility. For tie-breaking methods, however, the attenuation appears more consistent with random error. For instance, Ferrie (1996) with common names and ties and Soundex shows a 69 percent Type I error rate and the intergenerational elasticity estimate is 0.11 in Figure 7A.

Figure 7C and Figure 7D directly examine the effects of incorrect matches by plotting $\widehat{\pi}^{*}$, or the estimated elasticity for the "true" links (plotted as o with 95-percent confidence intervals) and $\widehat{\pi}^{\mathrm{i}}$, or the estimated elasticity for the "false” links (plotted as $\times$ ) from separate regressions. Without the incorrect links, the estimates of the intergenerational income elasticity are very similar across groups at around 0.23 without weights (Figure 7C) and 0.23 with inverse propensity-score weights (Figure 7D). The comparability of

indicator variable for presence of middle name, length of first, middle, and last name, polynomials in day of birth, polynomials in age, an index for first name commonness, an index for last name commonness, number of siblings, an indicator variable for presence of siblings, and the length of own name as well as father's and mother's names. We then use the estimated propensity of being linked, $P_{i}\left(L_{i}=1 \mid \boldsymbol{X}_{\boldsymbol{i}}\right)$, for each method and reweight observations by $\left(1-P_{i}\left(L_{i}=1 \mid \boldsymbol{X}_{\boldsymbol{i}}\right)\right) / P_{i}\left(L_{i}=1 \mid \boldsymbol{X}_{\boldsymbol{i}}\right) * q /(1-q)$, where $q$ is the share of records that are linked. Distributions of inverse propensity score weights are plotted in Appendix E. 
unweighted estimates is especially striking given how different in size and representativeness the samples are. For instance, the number of true links varies from around 482 for Ferrie 1996 (Soundex) to 1,600 when using exact ties Ferrie 1996 (Soundex), but the unweighted intergenerational income elasticities are estimated to be 0.22 and 0.23 , respectively. Consistent with Type I errors introducing attenuation, $\widehat{\pi}^{\mathrm{i}}$ tends to be smaller than $\widehat{\pi}^{*}$ across methods. And, consistent with the observations about the magnitudes above, the unweighted estimated intergenerational elasticities for the imputed links for Ferrie 1996 (Name) are 0.15 and only 0.05 for Abramitzky, Boustan, and Eriksson (2014) (Soundex) and 0.05 for Ferrie 1996 with common names and ties (Soundex)—a statistical zero in the latter two cases. On the other hand, the correlation of incorrect links for Feigenbaum (2016) is very high, which shows how the regression-based classification system selects links with a very high correlation to the true link in this setting-even when incorrect. In short, the inclusion of imputed links appears to have large effects on OLS estimates of the intergenerational income elasticities, biasing them toward zero in most cases. After purging incorrect links, reweighting the linked sample to resemble the set of birth certificates has a minimal effect on the estimates.

\section{LESSONS FOR HISTORICAL RECORD LINKING}

New large-scale linked data hold the potential to shift the frontier of knowledge. The need for reliable linking methods for U.S. Census data is especially high, where the linking variables in public data tend to be more limited than in modern administrative data and relative to records in other countries. Using different U.S. samples, this paper documents how linking errors could have large effects on scientific conclusions and policy inferences. Not only are linked samples not representative, but existing algorithms yield high rates of false matches. Moreover, the incidence of false matches are systematically related to baseline sample characteristics, suggesting that linking-induced measurement error may introduce complicated forms of bias into analyses. Our case study shows that linking algorithms may severely attenuate estimates of intergenerational income elasticities.

The variability in our estimates across datasets implies that it is difficult to diagnose how much linking assumptions matter for other records. Nevertheless, our results suggest that reducing false matches 
and choosing methods that generate false matches more highly correlated with the truth are crucial for improving inferences with linked data—even when reducing Type I errors results in higher rates of missed links.

An easy remedy for linking in richer data is to use more information to link records-especially continuous variables or those with many values (e.g., Social Security Numbers or exact dates of birth). In addition, higher quality information (e.g., administrative records rather than individual reports) will result in lower error rates than we document. For contexts with limited linking variables which are measured with error, systematic clerical review (e.g., LIFE-M) and genealogical methods (e.g., Early Indicators) generally attain lower error rates than machine algorithms. Because these methods are cost prohibitive for most projects, we draw on our findings to recommend several easy-to-implement and lower-cost changes to current practice.

First, we recommend careful examination of a sample of links resulting from automated algorithms. Applying close scrutiny to a sample of links allows researchers to diagnose and potentially remedy systematic problems with machine-linking algorithms arising for specific records or in a particular historical context. In fact, many of the links coded as incorrect in clerical review are easy to identify as such. These cases can be used to improve machine-linking algorithms.

Second, we recommend caution in linking phonetically cleaned names in deterministic algorithms or linking commonly occurring name-age combinations. Phonetic cleaning tends to remove meaningful variation in names that allows algorithms to make better links. Eliminating commonly occurring name-age combinations, like Ferrie’s (1996) approach of only linking uncommon names or Abramitzky, Boustan, and Eriksson (2014)'s robustness check using unique name-age combinations in a five-year window, substantially reduces the incidence of false matches. Together with reweighting, these restrictions also achieve results that are statistically indistinguishable from results with hand-linked data. In contrast, weighting name-age ties equally by the inverse of their empirical frequency incorporates information from a large number of false links and-in our case study—results in substantial attenuation. In addition, researchers may incorporate more information in the linking process to break ties and distinguish true links 
from close alternatives. One such example in historical data is middle name or middle initial.

Third, using even a small sample of clerically reviewed data to train a machine-learning algorithm (or applying the results of another researcher's model based on similar training data) can improve the quality of linked samples. Notably, even when these machine-methods make incorrect links, the correlation of these links with the truth appears to be much higher than for other algorithms in our setting. These errors, therefore, have less impact on inference. An additional feature of some machine linking methods is that they allow researchers to choose the importance of Type I and Type II errors, balancing the trade-off that to fit a particular application. Although Feigenbaum (1996) and Abramitzky, Mill, and Pérez (2018) choose a specific penalty for Type I and Type II errors, different parameter choices can drive Type I error rates lower while linking much of the sample correctly.

A fourth strategy for reducing Type I errors is to combine multiple methods and use the intersection of the links across sets—a form of ensemble machine learning in the spirit of "bagging” or "boosting." By construction, requiring links to be classified by more than one algorithm should tend to decrease match rates. But, to the extent that different methods make errors for different reasons, taking the set of common links helps avoid idiosyncratic reasons for errors. We illustrate the value of this approach in Figure 8 for our example of intergenerational elasticity, where we plot the Type I and Type II errors associated with the 131,071 possible combinations $\left(2^{17}-1\right)$ of the 17 algorithms in this paper for each dataset. Overall, combining methods drives down Type I error rates and increases Type II error rates. For example, when using LIFE-M data, combining two methods like Ferrie (1996) and Feigenbaum (2016) drives the Type I error rate to 10 percent-a substantial improvement over error rates for either method individually. Combining 12 methods achieves error rates as low as 6 percent, which is close to hand-linking.

Using combinations of methods may also improve inference. As shown in Figure 9A, across all combinations of methods, unweighted intergenerational elasticity estimates range from 0.11 to 0.24 (circle markers) and inverse-propensity-score reweighted estimates range from 0.13 to 0.24 (square markers). Based on an unweighted linear regression, a 10 percentage point increase in the Type I error rate tends to decrease the elasticity by 0.028 , whereas this number is 0.015 in the weighted regression. Interestingly, in 
both weighted and unweighted cases, the mean over all combinations yields the value to the elasticity obtained in the hand-linked LIFE-M sample. As in our intergenerational elasticity example using single methods, Figure 9B shows that eliminating the incorrect links yields an average intergenerational elasticity nearly identical to the hand-linked sample (0.22) whereas the average intergenerational elasticity estimates for the incorrect links are less than half that value (0.096). These findings hold even when considering only the most prominent matching algorithms.

Finally, after limiting linking errors as much as possible, we recommend using multiple record features to assess and improve sample representativeness. Survey methods for constructing weights and allocating values are easy to implement and have well-documented properties. Making greater use of common record features such as name length or other socio-demographic information also allows researchers to use survey research methods or, as is more common in economics (and used in this paper), construct inverse-propensity weights to reduce sample selection and improve representativeness in observed characteristics. ${ }^{34}$ The hope is that reweighting observed characteristics also improves balance in terms of unobserved characteristics, but there is no guarantee that it will. A close examination of what is referred to as the common support assumption also informs researchers about where more time-intensive genealogical or clerical review methods may increase the representation of hard-to-link groups.

Many discussions of inference with linked data implicitly or explicitly assume that the match rate is just as important to inference as match quality. Our findings suggest that the quality of inferences with linked data may be improved by putting less emphasis on increasing sample sizes (which in our analysis tend to be associated with higher rates of false matches) and more emphasis on increasing the share of correct links. That is, social scientists wishing to conduct inference on linked data might increase the weight they place on decreasing Type I error rates over increasing sample sizes (decreasing Type II error rates). In the parlance of machine learning, this would involve weighting precision more heavily than recall. Indeed, modern surveys such as the Panel Survey of Income Dynamics and the National Longitudinal Survey

${ }^{34}$ See Bailey, Cole, and Massey (2018) for a simple implementation description. 
demonstrate that much can be learned from high-quality small samples with summary statistics and weights

to describe and adjust for non-representativeness. Ultimately, increasing sample sizes for difficult-to-link subgroups (such as individuals with common names) will not likely be solved without more data or higher quality record features to disambiguate similar records. More research to uncover data to describe the groups underrepresented in linked samples will serve both to broaden knowledge about them and improve the ability of modern machine learning methods to link them.

\section{REFERENCES}

A'Hearn, Brian, Jörg Baten, and Dorothee Crayen. 2009. "Quantifying Quantitative Literacy: Age Heaping and the History of Human Capital." The Journal of Economic History 69 (3):783-808. doi: 10.1017/S0022050709001120.

Abowd, John M. 2017. "Large-scale Data Linkage from Multiple Sources: Methodology and Research Challenges." NBER Summer Institute Methods Lecture.

Abowd, John M., and Lars Vilhuber. 2005. "The Sensitivity of Economic Statistics to Coding Errors in Personal Identifiers." Journal of Business and Economic Statistics 23 (2):133-165.

Abramitzky, Ran, Leah Platt Boustan, and Katherine Eriksson. 2012. "Europe's Tired, Poor, and Huddled Masses: Self-Selection and Economic Outcomes in the Age of Mass Migration." American Economic Review 102 (5):1832-1856.

Abramitzky, Ran, Leah Platt Boustan, and Katherine Eriksson. 2013. "Have the Poor Always been Less Likely to Migrate? Evidence from Inheritance Practices during the Age of Mass Migration." Journal of Development Economics 102:2-14.

Abramitzky, Ran, Leah Platt Boustan, and Katherine Eriksson. 2014. "A Nation of Immigrants: Assimilation and Economic Outcomes in the Age of Mass Migration." Journal of Political Economy 122 (3):467-506.

Abramitzky, Ran, Roy Mill, and Santiago Pérez. 2018. "Linking Individuals Across Historical Sources: a Fully Automated Approach." National Bureau of Economic Research Working Paper Series No. 24324. doi: 10.3386/w24324.

Aizer, Anna, Shari Eli, Joseph Ferrie, and Adriana Lleras-Muney. 2016. "The Long Term Impact of Cash Transfers to Poor Families." American Economic Review 106 (4):935-971.

Antonie, Luiza, Kris Inwood, Daniel J. Lizotte, and J. Andrew Ross. 2014. "Tracking People Over Time in 19th Century Canada for Longitudinal Analysis." Machine Learning 95 (1):129-146.

Atack, Jeremy. 2004. "A Nineteenth-Century Resource for Agricultural History Research in the Twenty-First Century." Agricultural History 78 (4):389-412.

Atack, Jeremy, Fred Bateman, and Mary Eschelbach Gregson. 1992. "Matchmaker, Matchmaker, Make Me a Match." Historical Methods 25 (2):53-65.

Bailey, Amy Kate, Stewart E. Tolnay, E.M. Beck, and Jennifer D. Laird. 2011. "Targeting Lynch Victims: Social Marginality or Status Transgressions?" American Sociological Review 76 (3):412-436.

Bailey, Martha, Connor Cole, and Catherine G. Massey. 2018. "Simple Strategies for Improving Inference with Linked Data: A Case Study of the 1850-1930 IPUMS Linked Representative Historical Samples." University of Michigan Working Paper.

Bailey, Martha J. 2018. "Creating LIFE-M: The Longitudinal, Intergenerational Family Electronic Micro-Database." University of Michigan Working Paper.

Black, Sandra E., and Paul J. Devereux. 2011. "Recent Developments in Intergenerational Mobility." In Handbook of Labor Economics, edited by Card David and Ashenfelter Orley, 1487-1541. Amsterdam: Elsevier.

Bleakley, Hoyt , and Joseph Ferrie. 2016. "Shocking Behavior: Random Wealth in Antebellum Georgia and Human Capital Across Generations." Quarterly Journal of Economics 131 (3):1455-1495.

Bleakley, Hoyt , and Joseph P. Ferrie. 2013. "Up from Poverty? The 1832 Cherokee Land Lottery and the Long-run 
Distribution of Wealth." NBER Working Paper 19175.

Bleakley, Hoyt, and Joseph Ferrie. 2017. "Land Opening on the Georgia Frontier and the Coase Theorem in the Shortand Long- Run." http://www-personal.umich.edu/ hoytb/Bleakley_Ferrie_Farmsize.pdf.

Bogue, A. 1963. From Prairie to Corn Belt: Farming on the Illinois and Iowa Prairies in the Nineteenth Century. Chicago: University of Chicago Press.

Boustan, Leah Platt , Matthew E. Kahn, and Paul W. Rhode. 2012. "Moving to Higher Ground: Migration Response to Natural Disasters in the Early Twentieth Century." American Economic Review: Papers and Proceedings 102 (3):238-244.

Boustan, Leah Platt, and William Collins. 2014. "The Origins and Persistence of Black-White Differences in Women’s Labor Force Participation from the Civil War to the Present." In Human Capital and History: The American Record, edited by Leah Boustan, Carola Frydman and Robert A. Margo. Chicago, IL: University of Chicago Press.

Buckles, Karey S., and Daniel M. Hungerman. 2013. "Season of Birth and Later Outcomes: Old Questions, New Answers." Review of Economics and Statistics 95 (3):711-724.

Chetty, Raj, Nathaniel Hendren, Patrick Kline, Emmanuel Saez, and Nicholas Turner. 2014. "Is the United States Still a Land of Opportunity? Recent Trends in Intergenerational Mobility." American Economic Review 104 (5):141-47. doi: 10.1257/aer.104.5.141.

Christen, Peter, and Karl Goiser. 2007. Quality and Complexity Measures for Data Linkage and Deduplication. Vol. 43.

Collins, William J., and Marianne H. Wanamaker. 2014. "Selection and Economic Gains in the Great Migration of African Americans: New Evidence from Linked Census Data." American Economic Journal: Applied Economics 6 (1):220-252.

Collins, William J., and Marianne H. Wanamaker. 2015. "The Great Migration in Black and White: New Evidence on the Selection and Sorting of Southern Migrants." Journal of Economic History 75 (4):947-992.

Collins, William J., and Marianne H. Wanamaker. 2016. "Up from Slavery? African American Intergenerational Economic Mobility Since 1880." NBER Working Paper 23395.

Costa, Dora L., Heather DeSomer, Eric Hanss, Christopher Roudiez, Sven E. Wilson, and Noelle Yetter. 2017. "Union Army Veterans, All Grown Up." Historical Methods 50 (2):79-95.

Curti, Merle. 1959. The Making of an American Community: A Case Study of Democracy in a Frontier County. Stanford: Stanford University Press.

Dempster, A. P., N. M. Laird, and D. B. Rubin. 1977. "Maximum Likelihood from Incomplete Data via the EM Algorithm." Journal of the Royal Statistical Society. Series B (Methodological) 39 (1):1-38.

DiNardo, John, Nicole M. Fortin, and Thomas Lemieux. 1996. "Labor Market Institutions and the Distribution of Wages, 1973-1992: A Semiparametric Approach." Econometrica 64 (5):1001-1044.

Duncan, Otis Dudley. 1968. "Patterns of Occupational Mobility among Negro Men." Demography 5 (1):11-22.

Eli, Shari, Laura Salisbury, and Allison Shertzer. 2018. "Ideology and Migration after the American Civil War." Journal of Economic History 78 (3). doi: 10.1017/S0022050718000384.

Eriksson, Björn. 2016. "The Missing Links: Data Quality and Bias to Estimates of Social Mobility." www.fas.nus.edu.sg/cfpr/RC28/089.pdf. Accessed September 15, 2016.

Feigenbaum, James J. 2016. "A Machine Learning Approach to Census Record Linking." http://scholar.harvard.edu/files/jfeigenbaum/files/feigenbaum-censuslink.pdf?m=1423080976. Accessed March 28, 2016.

Fellegi, Ivan P., and Alan B. Sunter. 1969. "A Theory for Record Linkage." Journal of the American Statistical Association 64 (328):1183-1210. doi: 10.1080/01621459.1969.10501049.

Ferrie, Joseph P. 1996. "A New Sample of Males Linked from the 1850 Public Use Micro Sample of the Federal Census of Population to the 1860 Federal Census Manuscript Schedules." Historical Methods 29 (4):141156.

Goeken, Ronald, Tom Lynch, Yu Na Lee, Jacob Wellington, and Diana Magnuson. 2017.

Greene, William H. 2008. Econometric Analysis, 6th Edition. New York: Pearson.

Guest, A. M. 1987. "Notes from the National Panel Study: Linkage and Migration in the Late Nineteenth Century." Historical Methods 20 (2):63-77.

Hacker, J. David. 2010. "Decennial Life Tables for the White Population of the United States, 1790-1900." Historical Methods 43 (3):45-79.

Hacker, J. David. 2013. "New Estimates of Census Coverage in the United States, 1850-1930." Social Science History 37 (1):71-101.

Haider, Steven J., and Gary Solon. 2006. "Life-Cycle Variation in the Association between Current and Lifetime 
Earnings." American Economic Review 96 (4):1308-1320.

Heckman, James J., Hidehiko Ichimura, Jeff Smith, and Petra Todd. 1998. "Characterizing Selection Bias Using Experimental Data." Econometrica 66 (5):1017-1098.

Hornbeck, Richard, and Suresh Naidu. 2014. "When the Levee Breaks: Black Migration and Economic Development in the American South." American Economic Review 104 (3):963-990.

Horowitz, Joel L., and Charles F. Manski. 1995. "Identification and Robustness with Contaminated and Corrupted Data." Econometrica 63 (2):281-302. doi: 10.2307/2951627.

Hout, Michael, and Avery M. Guest. 2013. "Intergenerational Occupational Mobility in Great Britain and the United States since 1850: Comment." American Economic Review 103 (5):2021-40. doi: 10.1257/aer.103.5.2021.

Huber, P. J. . 1967. "The behavior of maximum likelihood estimates under nonstandard conditions." Proceedings of the Fifth Berkeley Symposium on Mathematical Statistics and Probability. 1:221-233.

Jaro, Matthew A. 1989. "Advances in Record Linking Methodology as Applied to Matching the 1985 Census of Tampa, Florida." Journal of the American Statistical Association 84 (406):414-420.

Kim, Gunky, and Raymond Chambers. 2012. "Regression Analysis under Probabilistic Multi-Linkage." Statistica Neerlandica 66 (1):64-79.

Lahiri, P., and Michael D. Larsen. 2005. "Regression Analysis with Linked Data." Journal of the American Statistical Association 100 (469):222-230.

Malin, J. 1935. "The Turnover of Farm Pouplation in Kansas." Kansas Historical 20:339-372.

Margo, Robert A. 2016. "Obama, Katrina, and the Persistence of Racial Inequality." Journal of Economic History 76 (2):301-341.

Massey, Catherine G. 2017. "Playing with matches: An assessment of accuracy in linked historical data." Historical Methods: A Journal of Quantitative and Interdisciplinary History:1-15. doi: 10.1080/01615440.2017.1288598.

Mazumder, Bhashkar. 2005. "Fortunate Sons: New Estimates of Intergenerational Mobility in the United States Using Social Security Earnings Data." Review of Economics and Statistics 87 (2):235-255. doi: 10.1162/0034653053970249.

Mazumder, Bhashkar. 2015. "Estimating the Intergenerational Elasticity and Rank Association in the U.S.: Overcoming the Current Limitations of Tax Data." Federal Reserve Bank of Chicago Working Paper.

Michelson, M. and Knoblock, C. A. 2006. "Learning Blocking Schemes for Record Linkage." Proceedings of the 21st National Conference on Artificial Intelligence AAAI-06.

Mill, Roy. 2013. "Record Linkage across Historical Datasets." Stanford University Dissertation. https://searchworks.stanford.edu/view/10232417.

Mill, Roy, and Luke C. Stein. 2016. "Race, Skin Color, and Economic Outcomes in Early Twentieth-Century America." Last Modified October 24, 2016. http://www.public.asu.edu/ lstein2/research/mill-steinskincolor.pdf.

Modalsli, Jorgen. 2017. "Intergenerational Mobility in Norway, 1865-2011." The Scandanavian Journal of Economics 119 (1):34-71. doi: 10.1111/sjoe.12196.

Mullainathan, Sendhil, and Jann Spiess. 2017. "Machine Learning: An Applied Econometric Approach." Journal of Economic Perspectives 31 (2):87-106.

National Office of Vital Statistics. 1948. State and Regional Life Tables, 1939-1941.

Nix, Emily, and Nancy Qian. 2015. "The Fluidity of Race: 'Passing' in the United States, 1880-1940." http://www.nber.org/papers/w20828.

Ruggles, Steven. 2006. "Linked Historical Censuses: A New Approach." History and Computing 14:213-224.

Ruggles, Steven. 2011. "Intergenerational Coredisence and Family Transitions in the United States, 1850-1880." Journal of Marriage and the Family 73 (1):138-148.

Ruggles, Steven, Catherine A. Fitch, and Evan Roberts. 2018. "Historical Census Record Linkage." Annual Review of Sociology 44.

Ruggles, Steven, Katie Genadek, Josiah Grover, and Matthew Sobek. 2015. Integrated Public Use Microdata Series (Version 6.0) [Machine-Readable database]. edited by University of Minnesota. Minneapolis: University of Minnesota.

Saperstein, Aliya, and Aaron Gullickson. 2013. "A Mulatto Escape Hatch? Examining Evidence of U.S. Racial and Social Mobility in the Jim Crow Era." Demography 50 (5):1921-1942.

Scheuren, Fritz, and William E. Winkler. 1993. "Regression analysis of data files that are computer matched." Survey methodology 19 (1):39-58.

Solon, Gary. 1992. "Intergenerational Income Mobility in the United States." American Economic Review 82 (3):393408. 
Solon, Gary. 1999. "Intergenerational Mobility in the Labor Market." In Handbook of Labor Economics, edited by Orley Ashenfelter and David Card, 1761-1800. Amsterdam: Elsevier.

Steckel, R. 1988. "Census Matching and Migration: A Research Strategy." Historical Methods 21:52-60.

Stephens, Melvin, Jr., and Takashi Unayama. forthcoming. "Estimating the Impacts of Program Benefits: Using Instrumental Variables with Underreported and Imputed Data." Review of Economics and Statistics.

Thernstrom, S. 1964. Poverty and Progress: Social Mobility in a Nineteenth Century City. Cambridge: Harvard University Press.

West, Kirsten K., and J. Gregory Robinson. 1999. "What do we know about the Undercount of Children?" U.S. Census Bureau Population Division Working Paper 39.

White, H. . 1980. "A heteroskedasticity-consistent covariance matrix estimator and a direct test for heteroskedasticity." Econometrica 48:817-830.

Winkler, William E. 2006. "Overview of Record Linkage and Current Research Directions." Research Report Series, Statistics 2006 (2).

Wisselgren, Maria J., Soren Edvinsson, Mats Berggren, and Maria Larsson. 2014. "Testing Methods of Record Linkage on Swedish Censuses." Historical Methods 47 (3):138-151.

Xie, Yue, and Alexandra Killewald. 2013. "Intergenerational Occupational Mobility in Britain and the U.S. since 1850: Comment." American Economic Review 103 (5):2003-2020.

Zimmerman, David J. 1992. "Regression Toward Mediocrity in Economic Stature." American Economic Review 82 (3):409-429. 
Figure 1. Examples of Common Linking Problems in Historical Samples

A. Albert Crock (Example of M1)

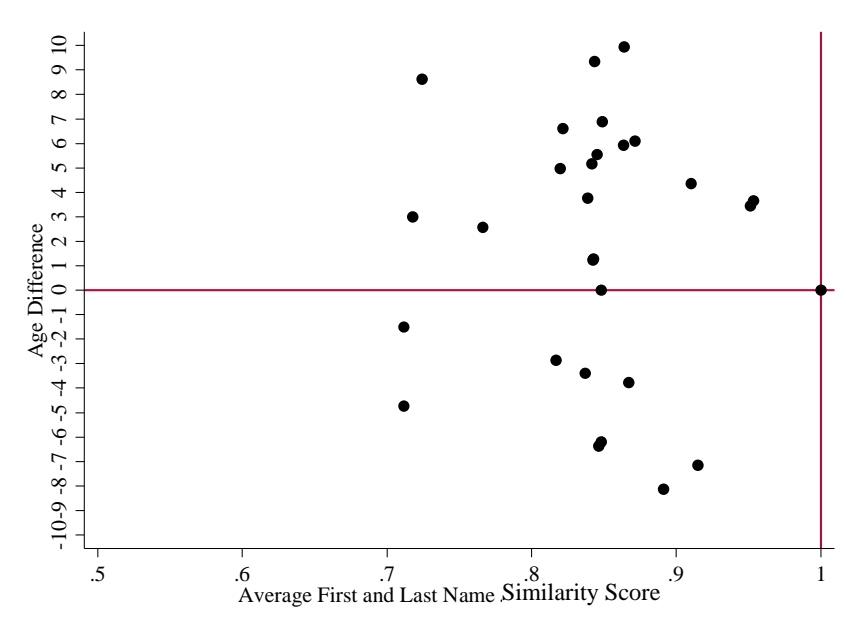

C. $\quad$ Arthur Smith (Example of M3)

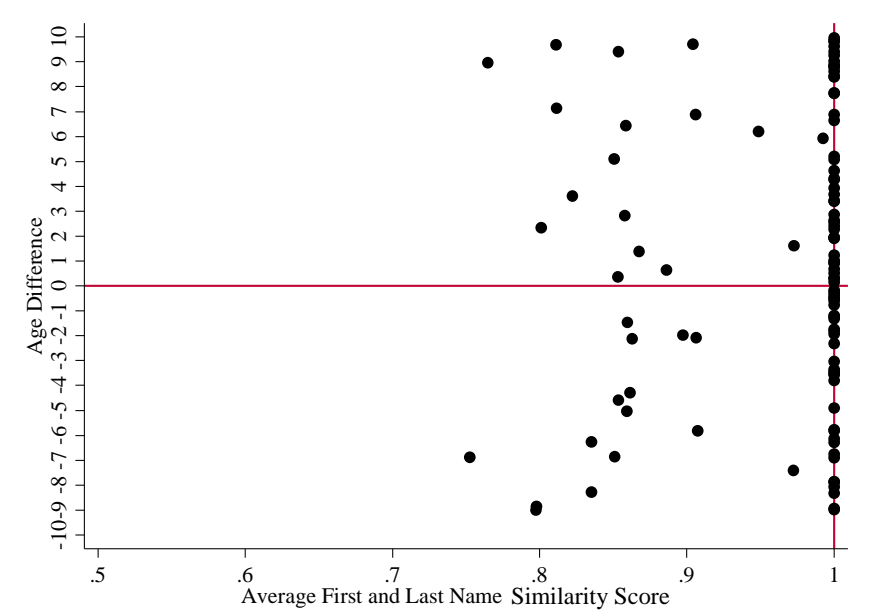

B. Raymond Bernaciak (Example of M2)

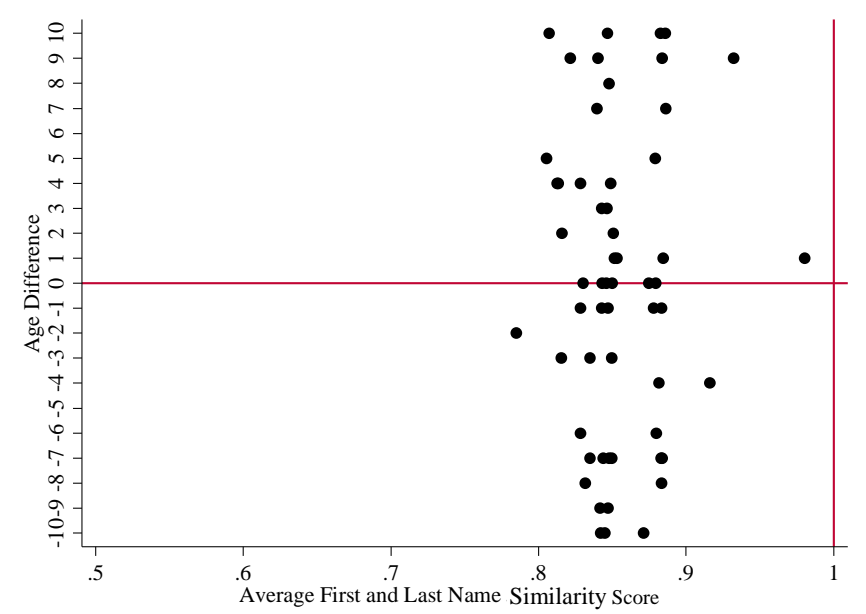

D. Charles Hall (Example of M4)

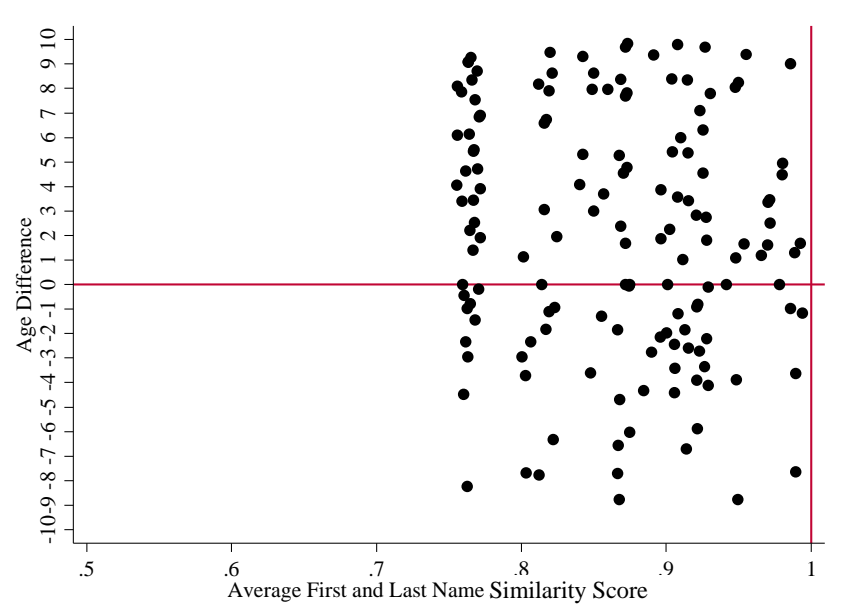


Figure 2. Match Rates and False Links for LIFE-M Hand-Linked Data and Selected Automated Linking Methods

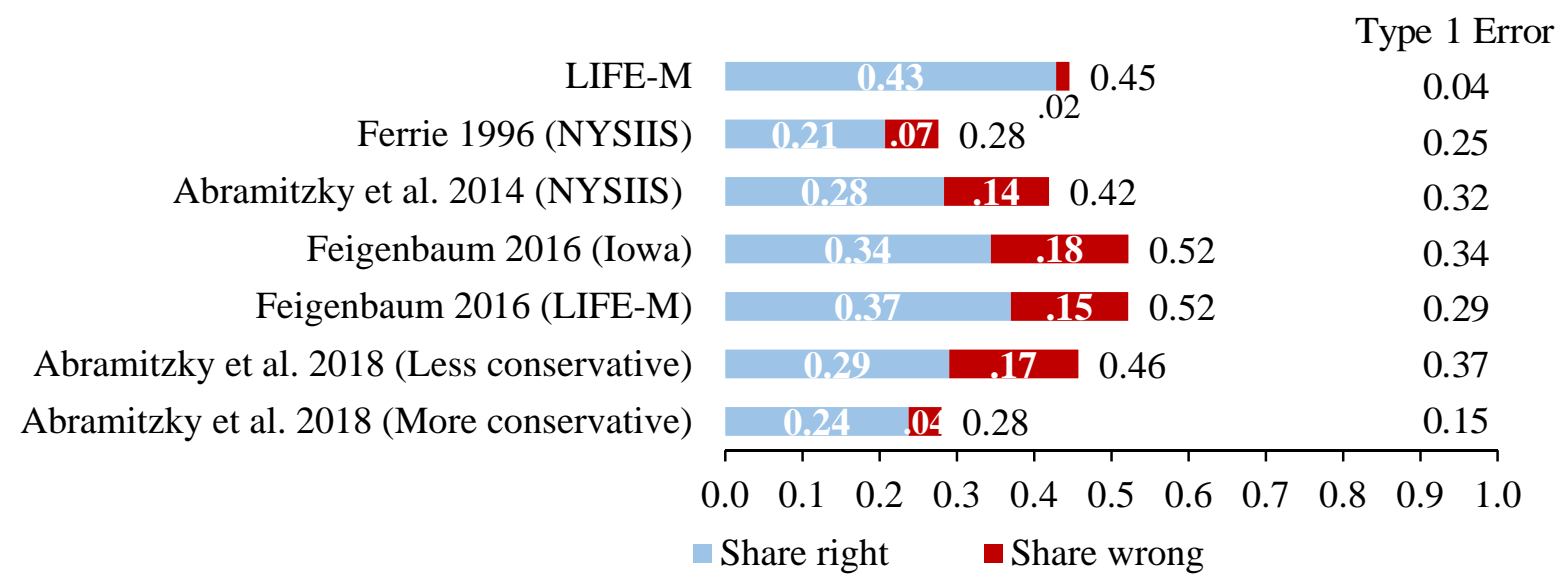

Notes: The bars show the performance of different algorithms linking LIFE-M boys to the 1940 Census. See text for details and Table 1 for numerical estimates.

Figure 3. Match Rates and False Links for Common Variations on Automated Linking Methods

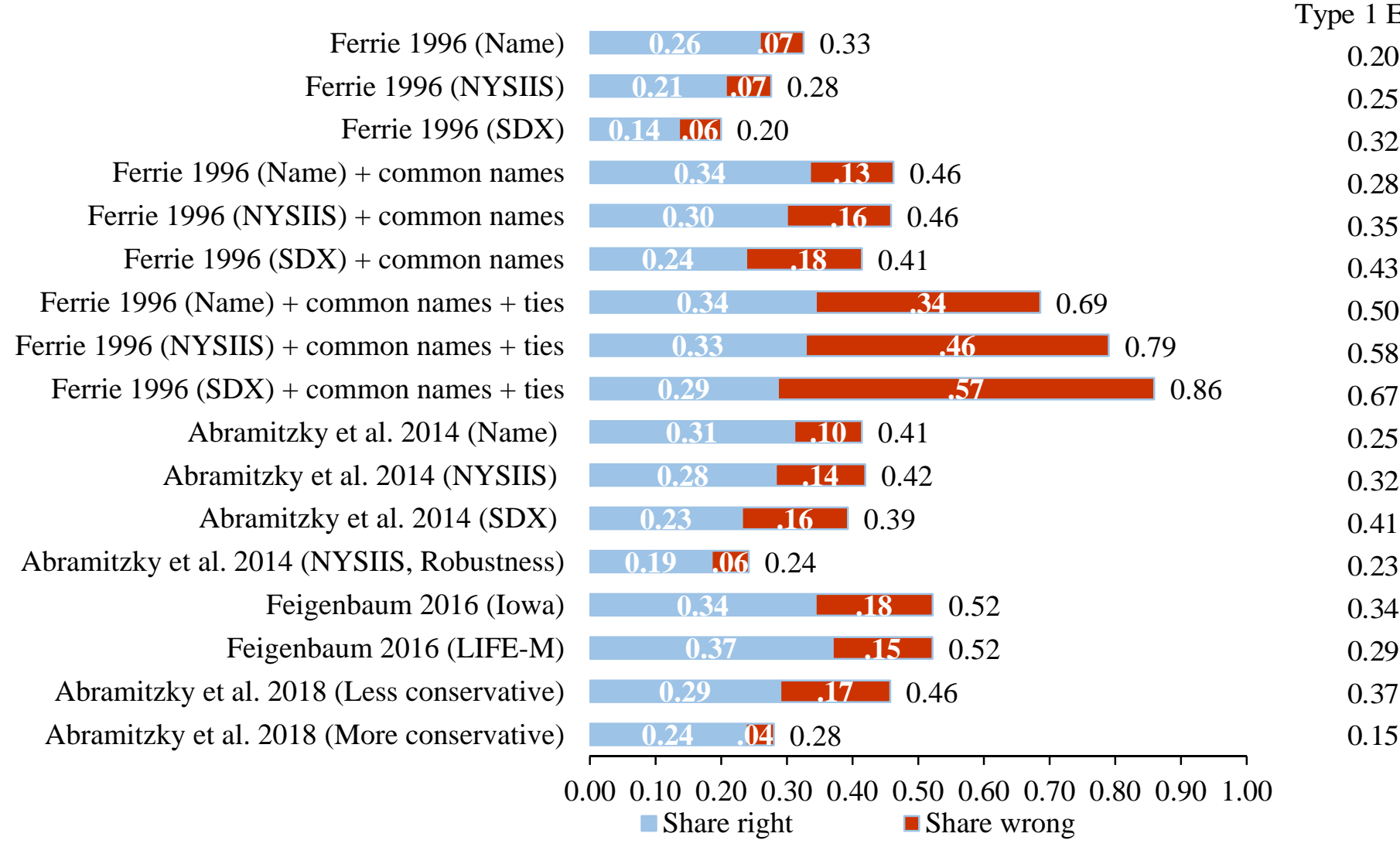

Notes: See Figure 2 notes. 
Figure 4. Share of Incorrect Links (Type I Error Rate) by Method and Dataset

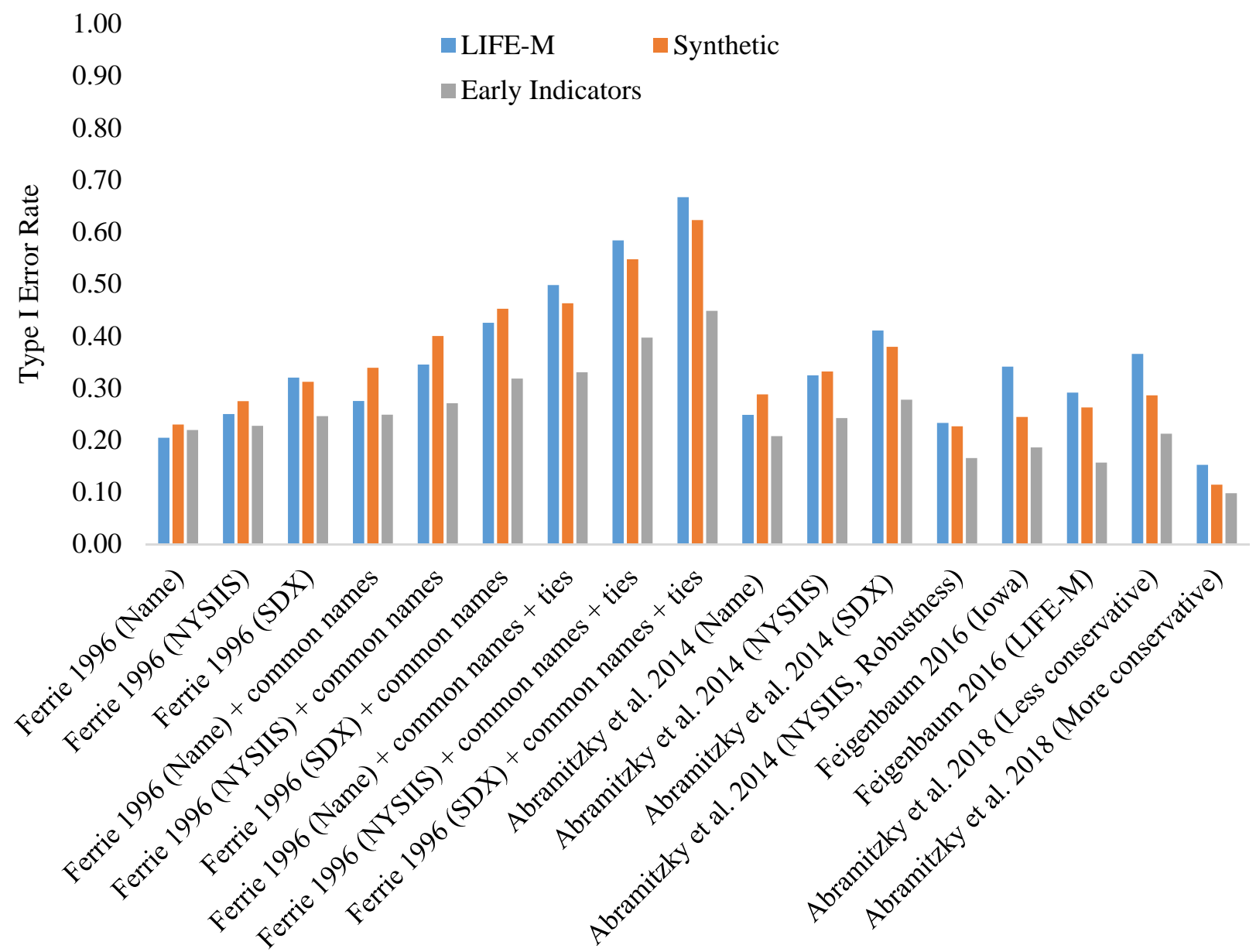

Notes: See Figure 2 notes and Table 1 for numerical estimates. 
Figure 5. Type I vs. Type II Error Rates by Method and Dataset

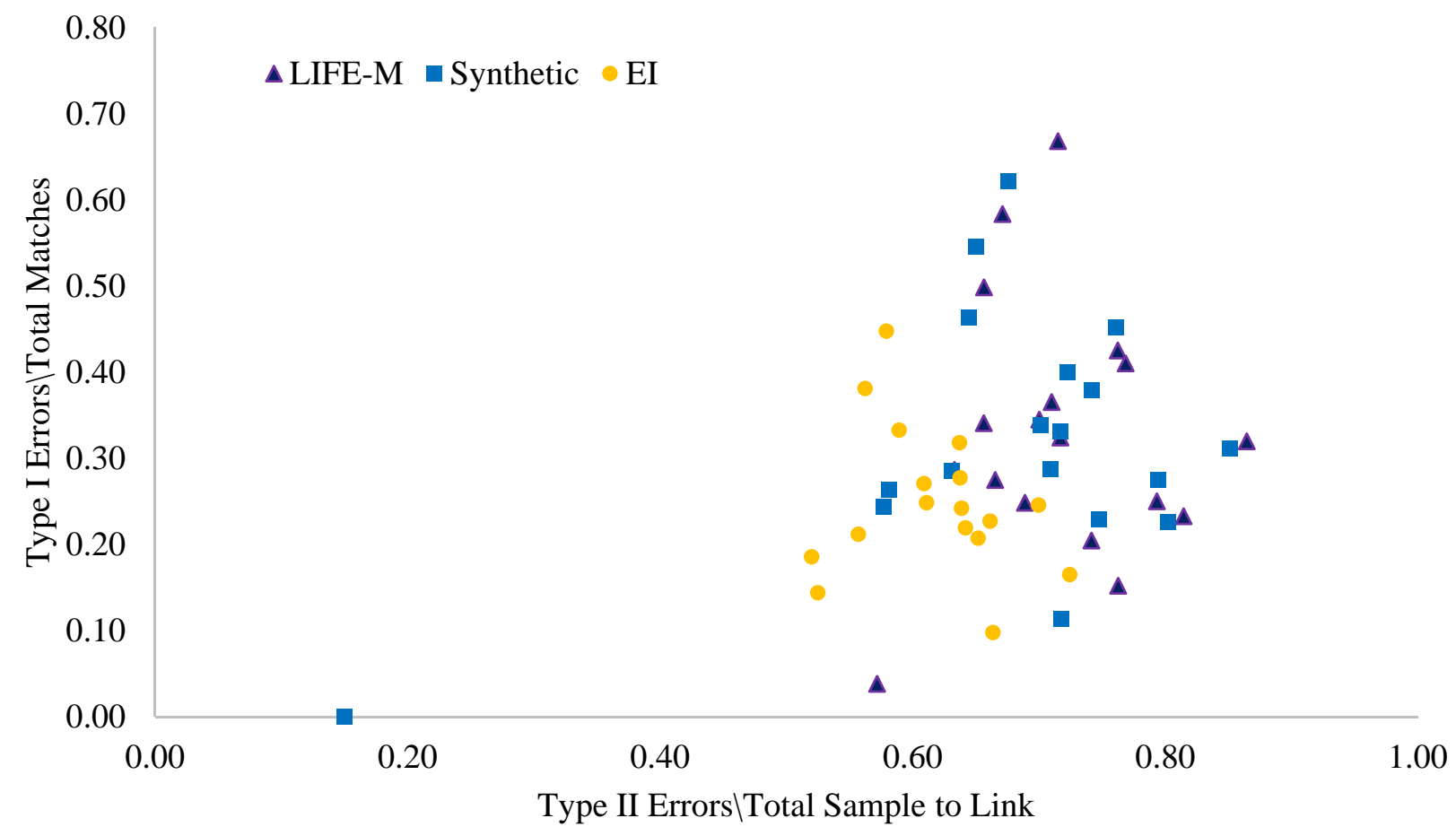

Notes: Points plot Type I and Type II error rates using different algorithms and data in Table 2. 


\section{Figure 6. A Comparison of Method Performance in Sample-to-Population and Population-to-}

Population Linking

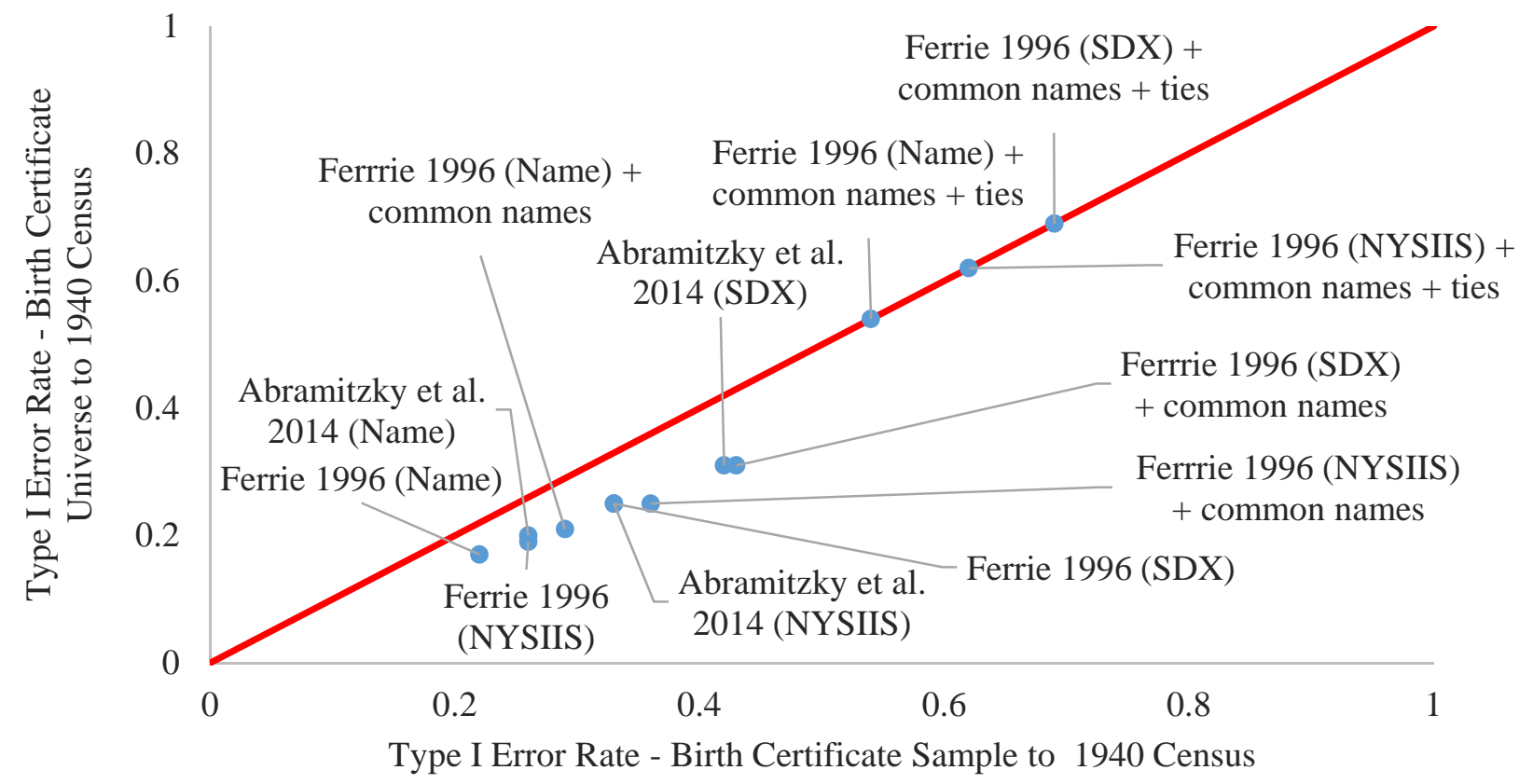

Notes: The y-axis plots the Type I error rate implied by linking birth certificates for all boys in the same cohorts as the Ohio and North Carolina LIFE-M sample to the 1940 Census using different automated methods. The x-axis plots the Type I error rate implied by linking the LIFE-M sample of boys in the Ohio and North Carolina birth certificates to the 1940 Census using different automated methods. 
Figure 7. Intergenerational Income Elasticity Estimates

A. Unweighted Linked Samples

LIFE-M
Ferrie 1996 (Name)
Ferrie 1996 (NYSIIs)
Ferrie 1996 (SDX)

Ferrie 1996 (Name) + common names Ferrie 1996 (NYSIIS) + common names

Ferrie $1996(\mathrm{SDX})+$ common names

Ferrie 1996 (Name) + common names + ties Ferrie 1996 (NYSIIS) + common names + ties

Ferrie $1996($ SDX) + common names + ties Abramitzky et al. 2014 (Name) Abramitzky et al. 2014 (NYSIIS)

Abramitzky et al. 2014 (SDX)

Abramitzky et al. 2014 (NYSIIS, Robustness) Feigenbaum 2016 (Iowa)

Feigenbaum 2016 (LIFE-M) Abramitzky et al. 2018 (Less conservative) Abramitzky et al. 2018 (More conservative)

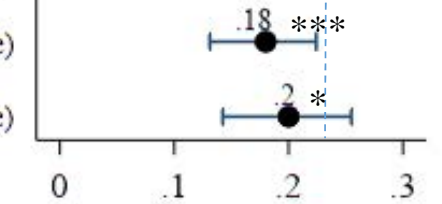

B. Inverse Propensity-Score Weighted Linked Samples

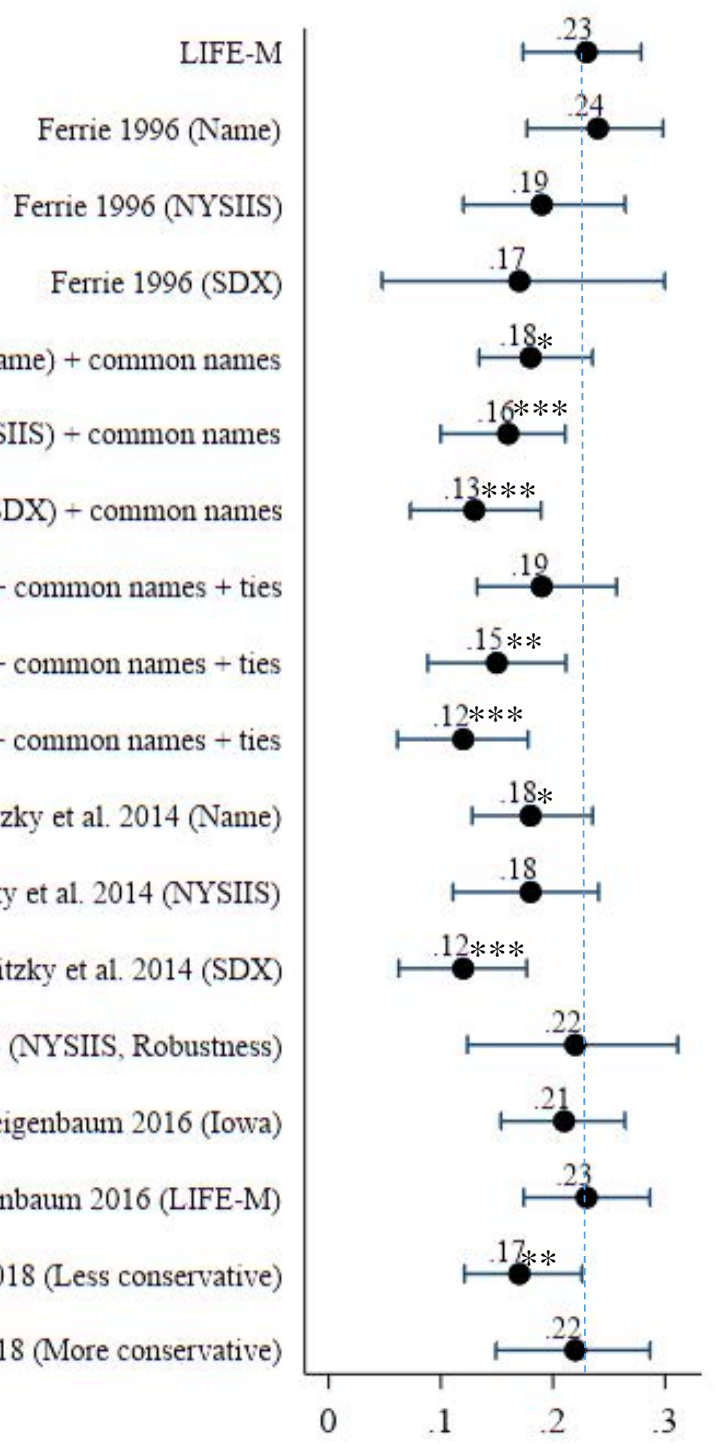




\section{LIFE-M Ferrie 1996 (Name) \\ Ferrie 1996 (NYSIIS) \\ Ferrie 1996 (SDX)}

Ferrie 1996 (Name) + common names

Ferrie 1996 (NYSIIS) + common names

Ferrie $1996(\mathrm{SDX})+$ common names

Ferrie 1996 (Name) + common names + ties

Ferrie 1996 (NYSIIS) + common names + ties

Ferrie $1996($ SDX $)+$ common names + ties

Abramitzky et al. 2014 (Name)

Abramitzky et al. 2014 (NYSIIS)

Abramitzky et al. 2014 (SDX)

Abramitzky et al. 2014 (NYSIIS, Robustness)

Feigenbaum 2016 (Iowa)

Feigenbaum 2016 (LIFE-M)

Abramitzky et al. 2018 (Less conservative)

Abramitzky et al. 2018 (More conservative)

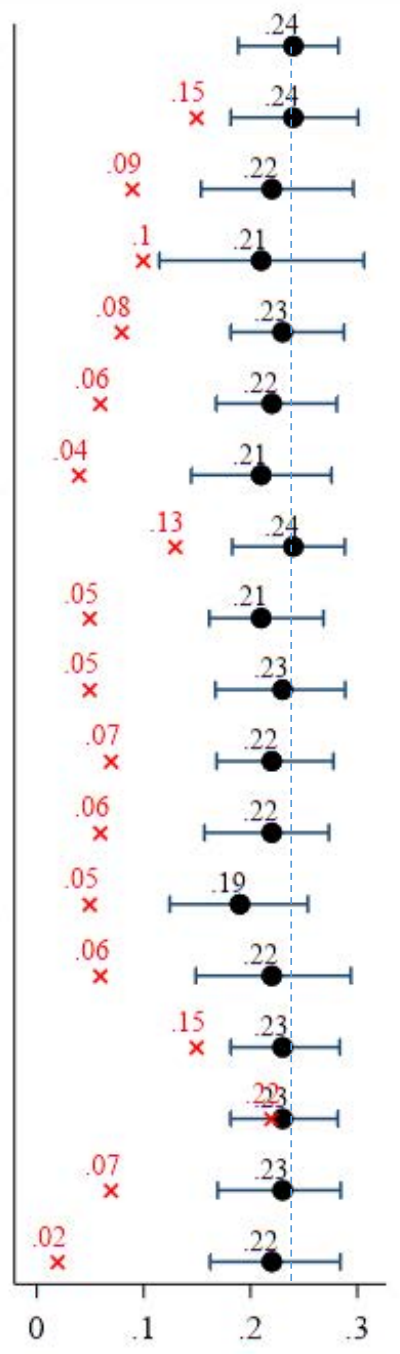

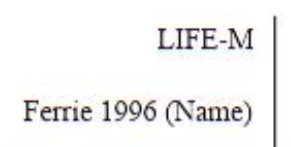

Ferrie 1996 (NYSIIS)

Ferrie 1996 (SDX)

Ferrie 1996 (Name) + common names

Ferrie 1996 (NYSIIS) + common names

Ferrie $1996(\mathrm{SDX})+$ common names

Ferrie 1996 (Name) + common names + ties

Ferrie 1996 (NYSIIS) + common names + ties

Ferrie $1996(\mathrm{SDX})+$ common names + ties Abramitzky et al. 2014 (Name) Abramitzky et al. 2014 (NYSIIS)

Abramitzky et al. 2014 (SDX)

Abramitzky et al. 2014 (NYSIIS, Robustness)

Feigenbaum 2016 (Iowa)

Feigenbaum 2016 (LIFE-M)

Abramitzky et al. 2018 (Less conservative)

Abramitzky et al. 2018 (More conservative)

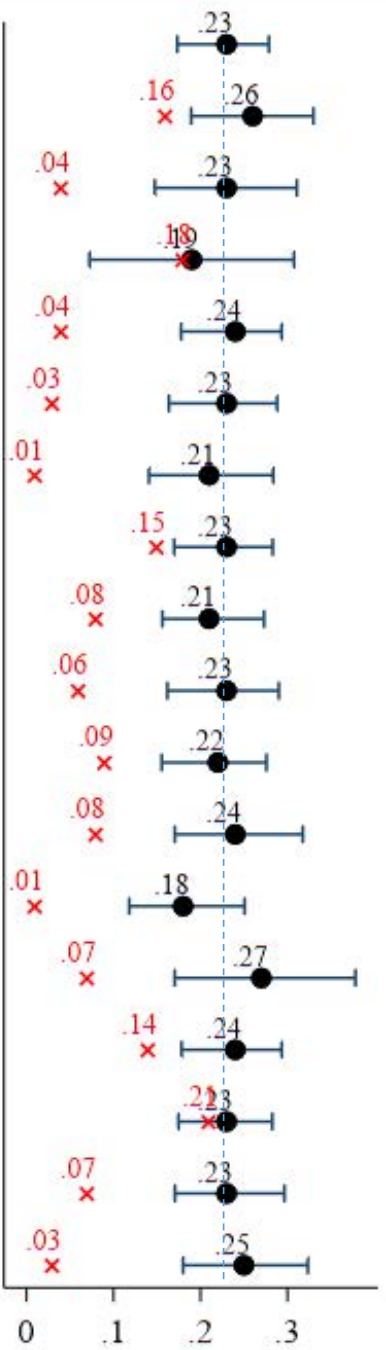

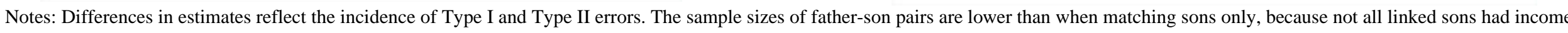

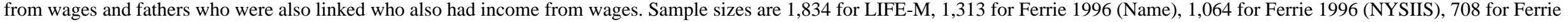

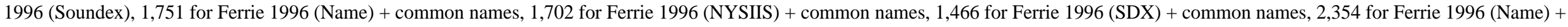

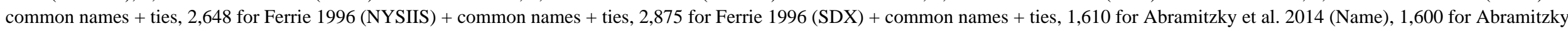

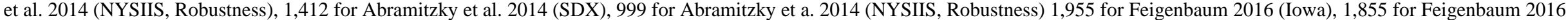

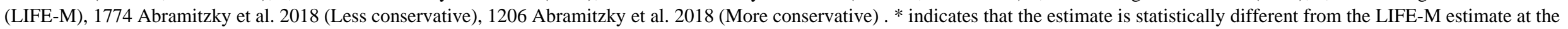
10 -percent, ${ }^{* *}$ at the 5 -percent, and ${ }^{* * *}$ at the 1 -percent levels. 
Figure 8. Type I vs. Type II Error Rates for Different Combinations of Methods and Dataset

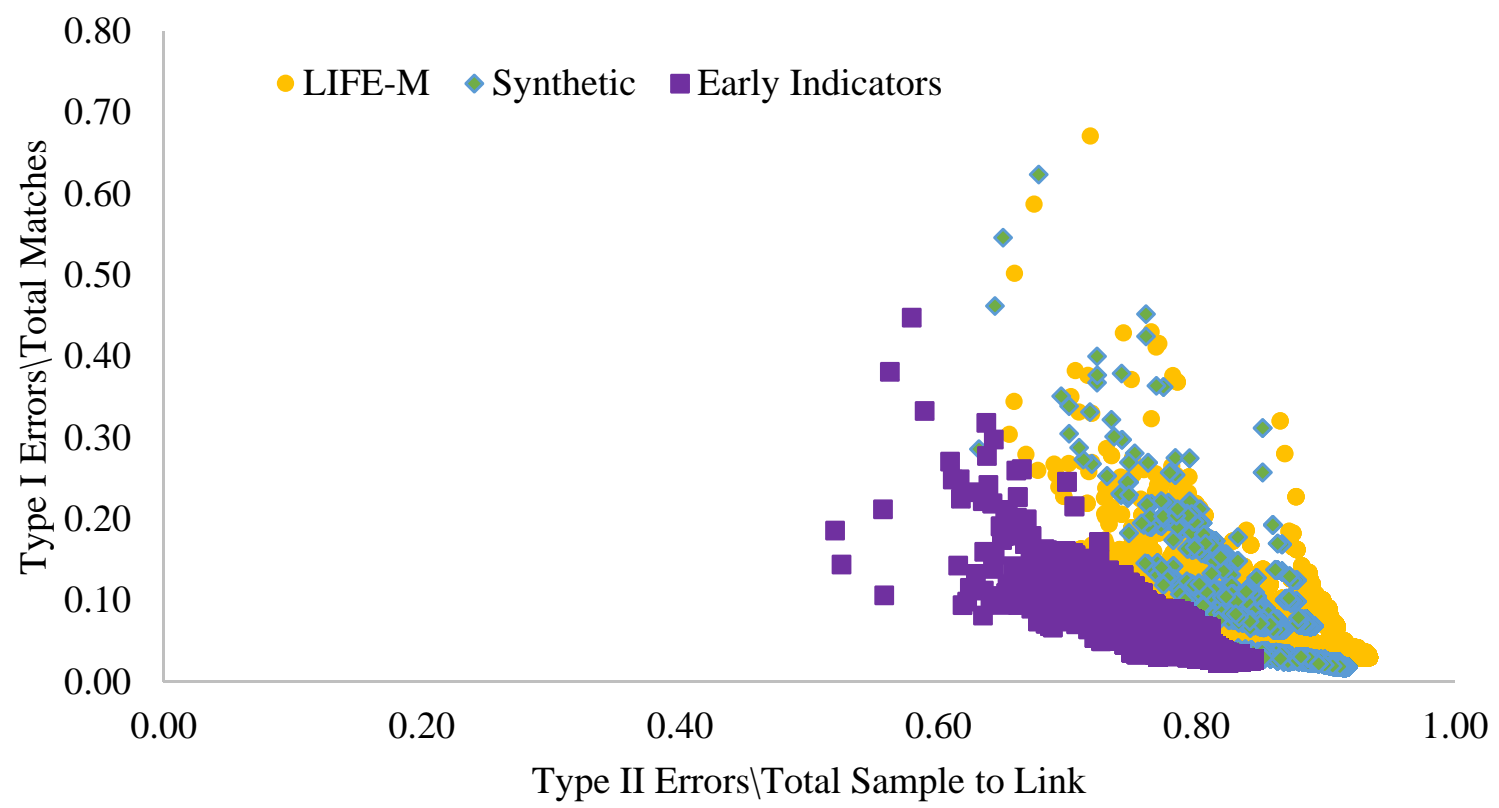

Notes: Each point represents the Type I and Type II error rate for 131,071 different combinations of the 17 methods considered in this paper by dataset. 


\section{Figure 9. Intergenerational Income Elasticity Estimates across Method Combinations}

\section{A. Unweighted and Weighted Intergenerational Income Elasticity Estimates}

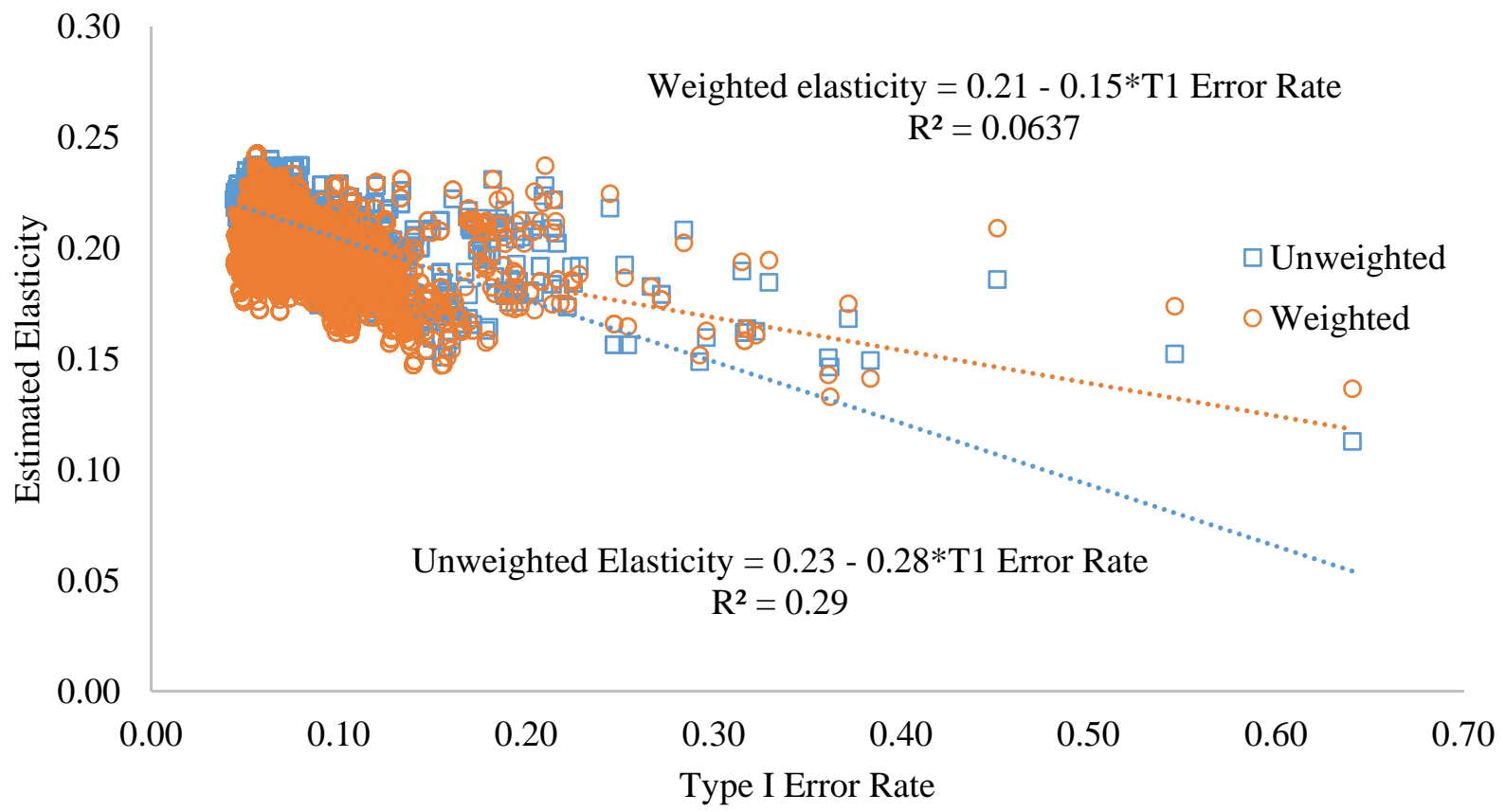

B. Intergenerational Income Elasticity Estimates using Correct versus Incorrect Links

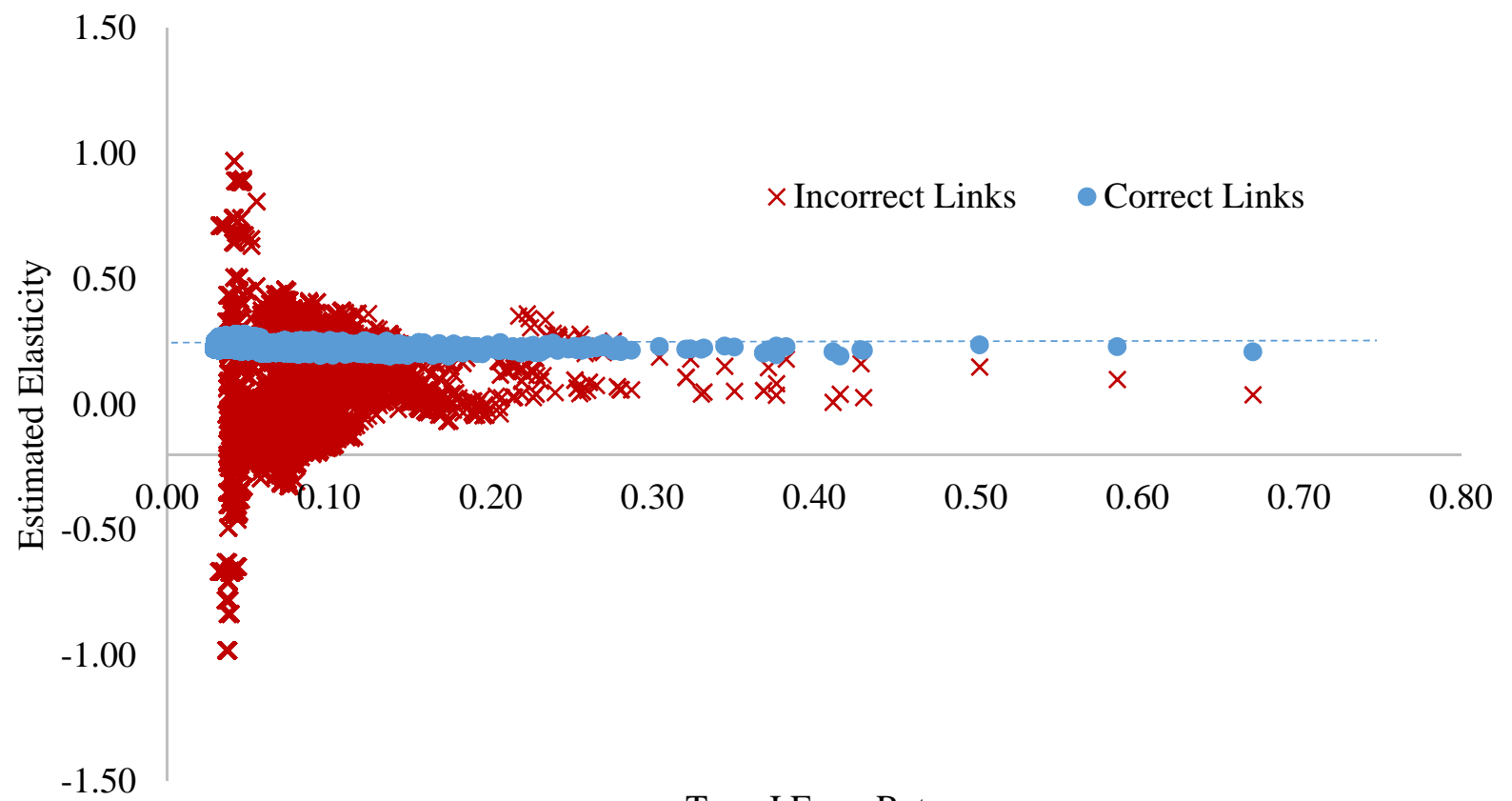

Type I Error Rate

Notes: Each point represents intergenerational elasticity estimate plotted against the Type I error rate for one of the 131,071 different combinations of the 17 methods considered in this paper. Panel A pools all links and panel B plots estimates separately for correct and incorrect links. See also Figure 6 notes. 
Table 1. Summary of Performance of Prominent Linking Methods, by Algorithm and Dataset

\begin{tabular}{|c|c|c|c|c|c|c|c|c|c|}
\hline & \multicolumn{3}{|c|}{ A. Match Rates } & \multicolumn{3}{|c|}{$\begin{array}{l}\text { B. Type I Error Rate (False } \\
\text { Links) }\end{array}$} & \multicolumn{3}{|c|}{$\begin{array}{l}\text { C. Type II Error Rate } \\
\text { (Missed links) }\end{array}$} \\
\hline & $\begin{array}{l}\text { LIFE- } \\
\mathrm{M}\end{array}$ & Synthetic & EI & LIFE-M & Synthetic & EI & LIFE-M & Synthetic & EI \\
\hline Hand-links or Synthetic Data & 0.45 & 0.85 & 1.00 & 0.04 & 0.00 & 0.00 & 0.57 & 0.15 & 0.00 \\
\hline Ferrie 1996 & 0.28 & 0.28 & 0.44 & 0.25 & 0.27 & 0.23 & 0.79 & 0.79 & 0.66 \\
\hline Abramitzky et al. 2014 & 0.42 & 0.42 & 0.48 & 0.32 & 0.33 & 0.24 & 0.72 & 0.72 & 0.64 \\
\hline Feigenbaum 2016 (Iowa coefficients) & 0.52 & 0.56 & 0.59 & 0.34 & 0.24 & 0.19 & 0.66 & 0.58 & 0.52 \\
\hline Feigenbaum 2016 (Estimated coefficients) & 0.52 & 0.57 & 0.57 & 0.29 & 0.26 & 0.14 & 0.63 & 0.58 & 0.52 \\
\hline Abramitzky et al. 2018 (Less conservative) & 0.46 & 0.52 & 0.56 & 0.37 & 0.29 & 0.21 & 0.71 & 0.63 & 0.56 \\
\hline Abramitzky et al. 2018 (More conservative) & 0.28 & 0.32 & 0.37 & 0.15 & 0.11 & 0.10 & 0.76 & 0.72 & 0.66 \\
\hline
\end{tabular}

Notes: EI stands for the "Early Indicators" data. Each estimate in the table is for a match rate, Type I error rate, or Type II error rate as described in text. These estimates are depicted in graphical form in Figures 2, 3 and 4. 
Table 2. Representativeness of Links Created by Prominent Linking Methods, by Algorithm and Dataset

\begin{tabular}{lccc}
\hline & & Synthetic & Early \\
& LIFE-M & Data & Indicators \\
\hline Ferrie 1996 (NYSIIS) & 445.9 & 277.5 & 38.2 \\
$p$-value & 0.00 & 0.00 & 0.00 \\
Abramitzky et al. 2014 (NYSIIS) & 457.0 & 387.2 & 12.7 \\
$p$-value & 0.00 & 0.00 & 0.24 \\
Feigenbaum 2016 (Iowa coef.) & 195.7 & 34.9 & 50.0 \\
p-value & 0.00 & 0.00 & 0.00 \\
Feigenbaum 2016 (Estimated coef.) & 334.9 & 62.2 & 44.0 \\
$p$-value & 0.00 & 0.00 & 0.00 \\
Abramitzky et al. 2018 (Less conservative) & 788.3 & 485.0 & 46.6 \\
$p$-value & 0.00 & 0.00 & 0.00 \\
Abramitzky et al. 2018 (More conservative) & 1350.0 & 673.0 & 51.4 \\
$p$-value & 0.00 & 0.00 & 0.00 \\
\hline Observations & 42,869 & 42,869 & 1,785 \\
\hline
\end{tabular}

Notes: Each estimate is a heteroskedasticity-robust Wald-test from a separate regression of a binary dependent variable (=1 for linked record) for samples described in the text. Relevant p-values are reported in italics. The covariates included in the LIFE-M sample and synthetic data are age, number of siblings, length of names of individuals and parents, fraction of siblings with misspelled parents' names, and an observation coming from Ohio. The covariates included in the Early Indicators data are age, currently married, foreign born, day of birth by year, literacy, length of first and last names, and foreign born status of parents. These sample sizes are slightly smaller due to missing values. See appendices for full regression results. 
Table 3. Randomness of False Links Created from Prominent Linking Methods, by Algorithm and Dataset

\begin{tabular}{lccc}
\hline & & Synthetic & Early \\
& LIFE-M & Data & Indicators \\
\hline Ferrie 1996 (NYSIIS) & 242.9 & 35.1 & 26.2 \\
$p$-value & 0.00 & 0.00 & 0.00 \\
Abramitzky et al. 2014 (NYSIIS) & 500.9 & 64.3 & 39.4 \\
$p$-value & 0.00 & 0.00 & 0.00 \\
Feigenbaum 2016 (Iowa coef.) & 1806.0 & 448.0 & 38.9 \\
$\quad$-value & 0.00 & 0.00 & 0.00 \\
Feigenbaum 2016 (Estimated coef.) & 1559.0 & 802.0 & 19.4 \\
$p$-value & 0.00 & 0.00 & 0.03 \\
Abramitzky et al. 2018 (Less conservative) & 559.3 & 112.9 & 43.0 \\
$p$-value & 0.00 & 0.00 & 0.00 \\
Abramitzky et al. 2018 (More conservative) & 139.8 & 17.4 & 18.3 \\
$p$-value & 0.00 & 0.03 & 0.05 \\
\hline
\end{tabular}

Notes: Each estimate is a heteroskedasticity-robust Wald-test from a separate regression of a binary dependent variable ( $=1$ for falsely linked record) for samples described in the text. Relevant p-values are reported in italics. The covariates included in the LIFE-M sample and synthetic data are age, number of siblings, length of names of individuals and parents, fraction of siblings with misspelled parents' names, and an observation coming from Ohio. The covariates included in the Early Indicators data are age, currently married, foreign born, day of birth by year, literacy, length of first and last names, and foreign born status of parents. See appendices for full regression results. 
Table 4. Summary of Algorithm Performance When Varying Assumptions

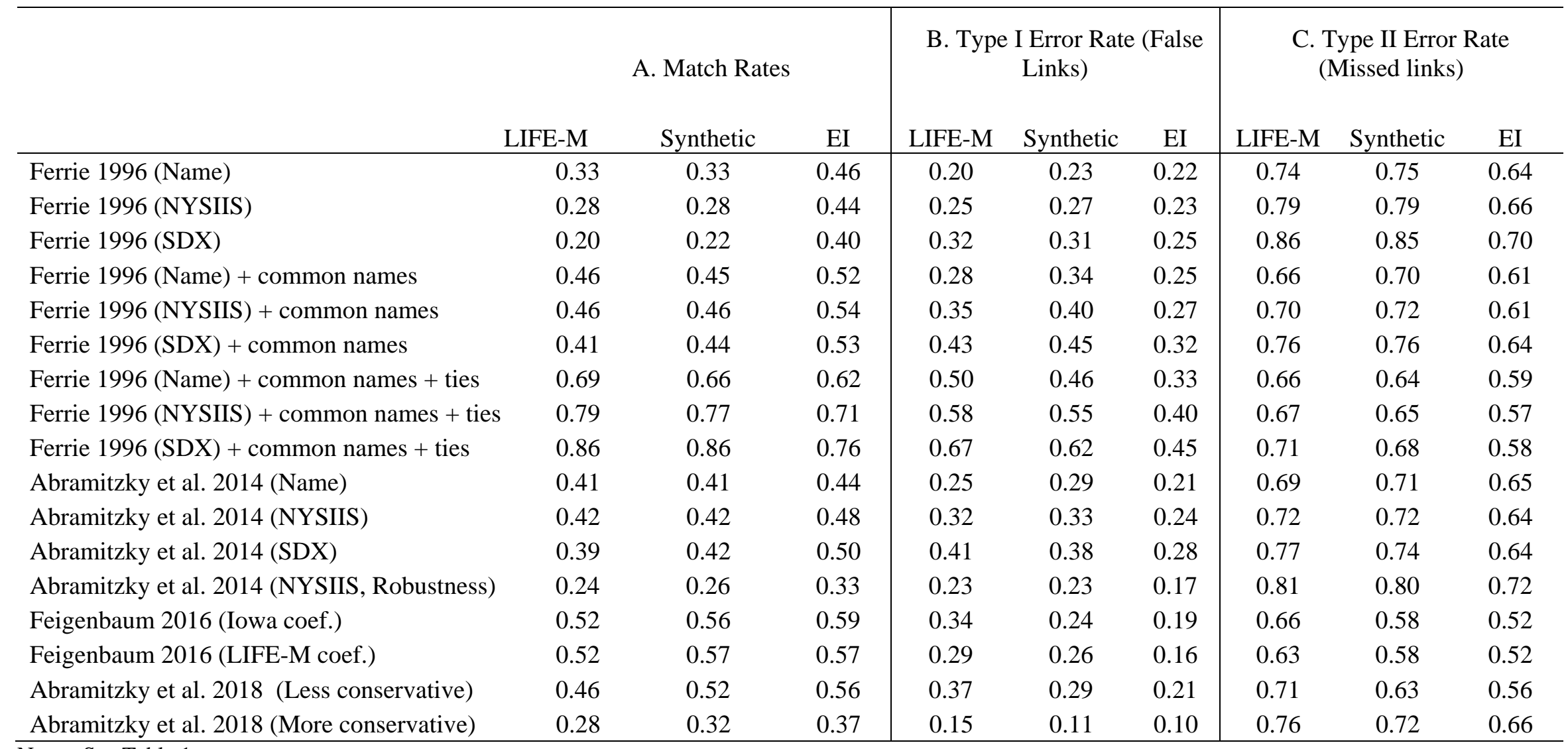


Table 5. Representativeness of Links When Varying Algorithm Assumptions

\begin{tabular}{|c|c|c|c|}
\hline & LIFE-M & $\begin{array}{c}\text { Synthetic } \\
\text { Data }\end{array}$ & $\begin{array}{c}\text { Early } \\
\text { Indicators }\end{array}$ \\
\hline Ferrie 1996 (Name) & 688.3 & 390.7 & 47.5 \\
\hline p-value & 0.00 & 0.00 & 0.00 \\
\hline Ferrie 1996 (NYSIIS) & 445.9 & 277.5 & 38.2 \\
\hline p-value & 0.00 & 0.00 & 0.00 \\
\hline Ferrie 1996 (SDX) & 130.6 & 71.1 & 57.0 \\
\hline p-value & 0.00 & 0.00 & 0.00 \\
\hline Ferrie 1996 (Name) + common names & 412.3 & 378.1 & 35.5 \\
\hline p-value & 0.00 & 0.00 & 0.00 \\
\hline Ferrie 1996 (NYSIIS) + common names & 402.6 & 447 & 16.0 \\
\hline p-value & 0.00 & 0.10 & 0.10 \\
\hline Ferrie $1996(\mathrm{SDX})+$ common names & 208.8 & 310.6 & 25.6 \\
\hline p-value & 0.00 & 0.00 & 0.00 \\
\hline Ferrie 1996 (Name) + common names + exact ties & 178.8 & 452.1 & 75.6 \\
\hline p-value & 0.00 & 0.00 & 0.00 \\
\hline Ferrie 1996 (NYSIIS) + common names + exact ties & 148.2 & 363.9 & 69.9 \\
\hline p-value & 0.00 & 0.00 & 0.00 \\
\hline Ferrie $1996(\mathrm{SDX})+$ common names + exact ties & 104.7 & 174.7 & 43.6 \\
\hline p-value & 0.00 & 0.00 & 0.00 \\
\hline Abramitzky et al. 2014 (Name) & 454.6 & 271.9 & 32.3 \\
\hline p-value & 0.00 & 0.00 & 0.00 \\
\hline Abramitzky et al. 2014 (NYSIIS) & 457.0 & 387.2 & 12.7 \\
\hline p-value & 0.00 & 0.00 & 0.24 \\
\hline Abramitzky et al. 2014 (SDX) & 255.7 & 257.8 & 17.1 \\
\hline p-value & 0.00 & 0.00 & 0.07 \\
\hline Abramitzky et al. 2014 (NYSIIS, Robustness) & 568.6 & 397.7 & 31.2 \\
\hline p-value & 0.00 & 0.00 & 0.00 \\
\hline Feigenbaum 2016 (Iowa coef.) & 195.7 & 34.9 & 50.0 \\
\hline p-value & 0.00 & 0.00 & 0.00 \\
\hline Feigenbaum 2016 (Estimated coef.) & 334.9 & 62.2 & 44.0 \\
\hline p-value & 0.00 & 0.00 & 0.00 \\
\hline Abramitzky et al. 2018 (Less conservative) & 788.3 & 485.0 & 46.6 \\
\hline p-value & 0.00 & 0.00 & 0.00 \\
\hline Abramitzky et al. 2018 (More conservative) & 1350.0 & 673.0 & 51.4 \\
\hline p-value & 0.00 & 0.00 & 0.00 \\
\hline Observations & 42,869 & 42,869 & 1,785 \\
\hline
\end{tabular}

Notes: See Table 2 notes. 
Table 6. Randomness of False Links When Varying Algorithm Assumptions

\begin{tabular}{|c|c|c|c|}
\hline & LIFE-M & $\begin{array}{c}\text { Synthetic } \\
\text { Data }\end{array}$ & $\begin{array}{c}\text { Early } \\
\text { Indicators }\end{array}$ \\
\hline Ferrie 1996 (Name) & 468.3 & 79.3 & 45.8 \\
\hline p-value & 0.00 & 0.00 & 0.00 \\
\hline Ferrie 1996 (NYSIIS) & 242.9 & 35.1 & 26.2 \\
\hline p-value & 0.00 & 0.00 & 0.00 \\
\hline Ferrie 1996 (SDX) & 81.5 & 0.9 & 17.5 \\
\hline p-value & 0.00 & 0.99 & 0.06 \\
\hline Ferrie 1996 (Name) + common names & 772.1 & 115.3 & 48.6 \\
\hline p-value & 0.00 & 0.00 & 0.00 \\
\hline Ferrie 1996 (NYSIIS) + common names & 429.0 & 64.4 & 39.2 \\
\hline p-value & 0.00 & 0.00 & 0.00 \\
\hline Ferrie $1996(\mathrm{SDX})$ + common names & 157.7 & 17.4 & 32.4 \\
\hline p-value & 0.00 & 0.03 & 0.00 \\
\hline Ferrie 1996 (Name) + common names + exact ties & 1859.0 & 466.2 & 60.1 \\
\hline p-value & 0.00 & 0.00 & 0.00 \\
\hline Ferrie 1996 (NYSIIS) + common names + exact ties & 1163.0 & 249.6 & 92.3 \\
\hline p-value & 0.00 & 0.00 & 0.00 \\
\hline Ferrie $1996($ SDX $)+$ common names + exact ties & 457.8 & 55.4 & 61.7 \\
\hline p-value & 0.00 & 0.00 & 0.00 \\
\hline Abramitzky et al. 2014 (Name) & 744.4 & 100.2 & 54.3 \\
\hline p-value & 0.00 & 0.00 & 0.00 \\
\hline Abramitzky et al. 2014 (NYSIIS) & 500.9 & 64.3 & 39.4 \\
\hline p-value & 0.00 & 0.00 & 0.00 \\
\hline Abramitzky et al. 2014 (SDX) & 223.2 & 41.7 & 28.6 \\
\hline p-value & 0.00 & 0.00 & 0.00 \\
\hline Abramitzky et al. 2014 (NYSIIS, Robustness) & 239 & 24.3 & 32.4 \\
\hline p-value & 0.00 & 0.00 & 0.00 \\
\hline Feigenbaum 2016 (Iowa coef.) & 1806.0 & 448 & 38.9 \\
\hline p-value & 0.00 & 0.00 & 0.00 \\
\hline Feigenbaum 2016 (Estimated coef.) & 1559.0 & 802 & 19.4 \\
\hline p-value & 0.00 & 0.00 & 0.03 \\
\hline Abramitzky et al. 2018 (Less conservative) & 559.3 & 112.9 & 43.0 \\
\hline p-value & 0.00 & 0.00 & 0.00 \\
\hline Abramitzky et al. 2018 (More conservative) & 139.8 & 17.4 & 18.3 \\
\hline p-value & 0.00 & 0.03 & 0.05 \\
\hline
\end{tabular}

Notes: See Table 3 notees. 
Table 7. How Middle Initials Could Reduce Errors in Linking in LIFE-M Data

\begin{tabular}{|c|c|c|c|c|c|c|c|}
\hline & $\begin{array}{c}\text { Table } 4 \\
\text { Match } \\
\text { Rate }\end{array}$ & $\begin{array}{l}(2) \\
\text { Table } 4 \\
\text { Type } \\
\text { Error } \\
\text { Rate }\end{array}$ & $\begin{array}{c}\text { (3) } \\
\text { Share } \\
\text { Matches with } \\
\text { Middle } \\
\text { Initials for } \\
\text { Both Records }\end{array}$ & $\begin{array}{l}\text { (4) } \\
\text { Share of } \\
\text { (3) with } \\
\text { Discordant } \\
\text { Middle } \\
\text { Initials }\end{array}$ & $\begin{array}{l}\text { (5) } \\
\text { Type I } \\
\text { Error Rate } \\
\text { in (4) }\end{array}$ & $\begin{array}{c}\text { Revised } \\
\text { Match Rate }\end{array}$ & $\begin{array}{c}\text { (7) } \\
\text { Revised } \\
\text { Type I } \\
\text { Error Rate }\end{array}$ \\
\hline Ferrie 1996 (Name) & 0.33 & 0.20 & 0.28 & 0.26 & 0.90 & 0.30 & 0.15 \\
\hline Ferrie 1996 (NYSIIS) & 0.28 & 0.25 & 0.26 & 0.27 & 0.91 & 0.26 & 0.20 \\
\hline Ferrie 1996 (SDX) & 0.20 & 0.32 & 0.24 & 0.30 & 0.93 & 0.19 & 0.27 \\
\hline Ferrie 1996 (Name) + common names & 0.46 & 0.28 & 0.29 & 0.35 & 0.94 & 0.42 & 0.20 \\
\hline Ferrie 1996 (NYSIIS) + common names & 0.46 & 0.35 & 0.27 & 0.37 & 0.95 & 0.41 & 0.28 \\
\hline Ferrie $1996($ SDX) + common names & 0.41 & 0.43 & 0.26 & 0.41 & 0.96 & 0.37 & 0.36 \\
\hline Ferrie 1996 (Name) + common names + exact ties & 0.69 & 0.50 & 0.30 & 0.44 & 0.97 & 0.60 & 0.43 \\
\hline Ferrie 1996 (NYSIIS) + common names + exact ties & 0.79 & 0.58 & 0.29 & 0.50 & 0.98 & 0.68 & 0.52 \\
\hline Ferrie $1996($ SDX $)+$ common names + exact ties & 0.86 & 0.67 & 0.28 & 0.57 & 0.98 & 0.72 & 0.61 \\
\hline Abramitzky et al. 2014 (Name) & 0.41 & 0.25 & 0.30 & 0.31 & 0.94 & 0.38 & 0.18 \\
\hline Abramitzky et al. 2014 (NYSIIS) & 0.42 & 0.32 & 0.27 & 0.33 & 0.94 & 0.38 & 0.26 \\
\hline Abramitzky et al. 2014 (SDX) & 0.39 & 0.41 & 0.27 & 0.39 & 0.96 & 0.35 & 0.35 \\
\hline Abramitzky et al. 2014 (NYSIIS, Robustness) & 0.24 & 0.23 & 0.26 & 0.26 & 0.89 & 0.23 & 0.18 \\
\hline Feigenbaum 2016 (Iowa) & 0.52 & 0.34 & 0.31 & 0.23 & 0.93 & 0.48 & 0.30 \\
\hline Feigenbaum 2016 (LIFEM) & 0.52 & 0.29 & 0.33 & 0.23 & 0.93 & 0.48 & 0.24 \\
\hline Abramitzky et al. 2018 (Less conservative) & 0.46 & 0.37 & 0.29 & 0.34 & 0.95 & 0.41 & 0.30 \\
\hline Abramitzky et al. 2018 (More conservative) & 0.28 & 0.15 & 0.29 & 0.20 & 0.87 & 0.26 & 0.11 \\
\hline
\end{tabular}

Notes: This table uses the LIFE-M data to evaluate changes in algorithm match rates and Type I error rates with the addition of middle initial. Column 7 computes match rates after dropping links with discordant middle initials. Column 8 computes revised Type I error rates by dropping links with discordant middle initials. See text for details. 
Table 8. How Using Race Could Reduce Errors in Linking in LIFE-M Data

\begin{tabular}{lccccc}
\hline & $(1)$ & $(2)$ & $(3)$ & $(4)$ & $(5)$ \\
& & Table & Share Matches - & & \\
& Table & 4 & 1940 Race & & \\
& 4 & Type I & Variables & Revised & Revised \\
& Match & Error & Different than & Match & Type I \\
& Rate & Rate & LIFE-M & Rate & Error Rate \\
\hline Ferrie 1996 (Name) & 0.33 & 0.20 & 0.00 & 0.33 & 0.20 \\
Ferrie 1996 (NYSIIS) & 0.28 & 0.25 & 0.01 & 0.28 & 0.25 \\
Ferrie 1996 (SDX) & 0.20 & 0.32 & 0.01 & 0.20 & 0.31 \\
Ferrie 1996 (Name) + common names & 0.46 & 0.28 & 0.01 & 0.46 & 0.27 \\
Ferrie 1996 (NYSIIS) + common names & 0.46 & 0.35 & 0.01 & 0.46 & 0.34 \\
Ferrie 1996 (SDX) + common names & 0.41 & 0.43 & 0.02 & 0.41 & 0.42 \\
Ferrie 1996 (Name) + common names + exact ties & 0.69 & 0.50 & 0.01 & 0.69 & 0.49 \\
Ferrie 1996 (NYSIIS) + common names + exact ties & 0.79 & 0.58 & 0.03 & 0.79 & 0.58 \\
Ferrie 1996 (SDX) + common names + exact ties & 0.86 & 0.67 & 0.06 & 0.86 & 0.66 \\
Abramitzky et al. 2014 (Name) & 0.41 & 0.25 & 0.01 & 0.41 & 0.25 \\
Abramitzky et al. 2014 (NYSIIS) & 0.42 & 0.32 & 0.01 & 0.42 & 0.32 \\
Abramitzky et al. 2014 (SDX) & 0.39 & 0.41 & 0.02 & 0.39 & 0.40 \\
Abramitzky et al. 2014 (NYSIIS, Robustness) & 0.24 & 0.23 & 0.01 & 0.24 & 0.23 \\
Feigenbaum 2016 (Iowa) & 0.52 & 0.34 & 0.01 & 0.52 & 0.34 \\
Feigenbaum 2016 (LIFEM) & 0.52 & 0.29 & 0.00 & 0.52 & 0.29 \\
Abramitzky et al. 2018 (Less conservative) & 0.46 & 0.37 & 0.02 & 0.46 & 0.36 \\
Abramitzky et al. 2018 (More conservative) & 0.28 & 0.15 & 0.00 & 0.28 & 0.15 \\
\hline
\end{tabular}

Notes: This table uses the LIFE-M to evaluate changes in linking rates with the addition of race. Column 4 computes match rates after dropping links with discordant race. Column 5 computes revised Type I error rates by dropping links with discordant race. See text for details.

\section{[Click here for Online Appendices]}

